\title{
Sensor Selection Based on Generalized Information Gain for Target Tracking in Large Sensor Networks
}

\author{
Xiaojing Shen, Member, IEEE, Pramod K. Varshney, Fellow, IEEE *
}

May 9, 2018

\begin{abstract}
In this paper, sensor selection problems for target tracking in large sensor networks with linear equality or inequality constraints are considered. First, we derive an equivalent Kalman filter for sensor selection, i.e., generalized information filter. Then, under a regularity condition, we prove that the multistage lookahead policy that minimizes either the final or the average estimation error covariances of next multiple time steps is equivalent to a myopic sensor selection policy that maximizes the trace of the generalized information gain at each time step. Moreover, when the measurement noises are uncorrelated between sensors, the optimal solution can be obtained analytically for sensor selection when constraints are temporally separable. When constraints are temporally inseparable, sensor selections can be obtained by approximately solving a linear programming problem so that the sensor selection problem for a large sensor network can be dealt with quickly. Although there is no guarantee that the gap between the performance of the chosen subset and the performance bound is always small, numerical examples suggest that the algorithm is near-optimal in many cases. Finally, when the measurement noises are correlated between sensors, the sensor selection problem with temporally inseparable constraints can be relaxed to a Boolean quadratic programming problem which can be efficiently solved by a Gaussian randomization procedure along with solving a semi-definite programming problem. Numerical examples show that the proposed method is much better than the method that ignores dependence of noises.
\end{abstract}

keywords: Sensor selection; generalized information gain; sensor networks, target tracking

*This work was supported in part by U.S. Air Force Office of Scientific Research (AFOSR) under Grants FA9550-10-1-0263 and FA9550-10-1-0458 and in part by the NNSF of China \# 61004138 and IRT1273.

${ }^{\dagger}$ Xiaojing Shen (corresponding author, shenxj@scu.edu.cn) and Pramod K. Varshney (varshney@syr.edu) are with the Department of Electrical Engineering and Computer Science, Syracuse University, NY, 13244, USA. Xiaojing Shen (shenxj@scu.edu.cn) is on leave from Department of Mathematics, Sichuan University, Chengdu, Sichuan 610064, China. 


\section{Introduction}

Over the past twenty years, advances in sensor technologies have led to the emergence of large numbers of low-cost sensing devices with a fair amount of computing and communication capabilities. Large sensor networks have attracted much attention both from theoretical and practical standpoints and have become a fast-growing research area. To efficiently manage large sensor networks, one typically designs a policy for determining the optimal sensor network performance and resource utilization at each time, within logical or budget constraints. The most comprehensive recent survey on sensor management is provided in the book [1]. Discussion on more advances in this area is available in the recent survey paper [2] and references therein. In this paper, we concentrate on sensor selection problems in which a subset of sensors are selected at each time instant while tracking a target that provides optimal performance-resource usage tradeoffs.

The sensor selection problem arises in various applications, including target tracking, e.g., [3, 4], robotics [5], and wireless networks [6]. Sensor selection for the target tracking problem will be considered here. In the literature, the sensor selection problem has been formulated for different dynamic systems. In [3], the state model was assumed to be deterministic without noise. A convex optimization procedure was developed based on a heuristic to solve the problem of selecting $k$ sensors from a set of $m$ sensors. Although no optimality guarantees could be provided for the solution, numerical experiments showed that it performed well. Another important contribution comes from the work reported in [4] where the state model was assumed random with noise and a general objective function of the sensor selection problem was transformed to a quadratic form by introducing the gain matrix as an additional decision variable. However, the resulting optimization problem cannot efficiently take advantage of the structure of the covariance of measurement noise such as it being a diagonal matrix in the uncorrelated case. In this paper, the sensor selection problem formulated by the use of the Moore-Penrose generalized inverse only relies on Boolean decision variables without introducing additional decision variables. The resulting optimization problem can efficiently take advantage of the structure of the measurement noise and obtain the optimal solution analytically. Many other excellent results on sensor selection for state estimation in different situations can be found in, e.g., $[7,8,9,10,11,12,13,14,15,16,17,18]$ and references therein.

Sensor management problems are often considered with different criteria and objectives. Representative approaches for sensor management include optimization of estimation error covariance [3, 4], Fisher information [9, 10], and entropy or mutual information [5, 11, 12, 13, 14, 15, 18, 19]. Various functions of

the estimation error covariance and Fisher information matrix, including their determinant and trace, have been used as reward functions for optimal sensor management. Several popular measures, including Rényi entropy, Kullback-Leibler (KL) divergence, and Hellinger-Battacharya distance, have been used for the calculation of information gain between two densities. In this paper, based on the Moore-Penrose generalized inverse, we will derive a closed-form expression of information gain for sensor selection called generalized information gain whose trace function is taken as the reward function for optimal sensor selection. When the measurement noises are assumed independent, the notion of information measure based on the information 
gain has been discussed in the literature, see [19].

In this paper, we consider the problem of state estimation for a linear dynamic system being monitored by multiple sensors. For sensor selection, we first derive an equivalent Kalman filter for sensor selection, i.e., generalized information filter. Then, under a regularity condition, we prove that the multistage lookahead policy that minimizes either the final or the average estimation error covariance of next $N$ time steps is equivalent to a myopic sensor selection policy that maximizes the trace of the generalized information gain at each time step. Thus, trace of the generalized information gain is defined as a measure of information that the selected sensors provide at each time step. Moreover, when the measurement noises are uncorrelated between sensors, the optimal solution can be obtained analytically when the constraints are temporally separable. When the constraints are temporally inseparable, the solution of the sensor selection problem can be obtained by approximately solving a linear program (LP) so that sensor selections for a large sensor network can be performed quickly. Although there is no guarantee that the gap between the performance of the chosen subset and the performance bound is always small, numerical examples suggest that the algorithm is nearoptimal in many cases. Finally, when the measurement noises are correlated between sensors, the sensor selection problem when the constraints are temporally inseparable can be relaxed to a Boolean quadratic programming (BQP) which can be efficiently solved by a Gaussian randomization procedure along with solving a semi-definite programming (SDP) problem which can be solved by interior-point methods [20]. Numerical examples show that the proposed method yields solutions that are much better than the method that ignores dependence.

The rest of the paper is organized as follows. Preliminaries are given in Section 2, where the generalized information filter for sensor selection and multistage sensor selection problems that minimize either the final or the average estimation error covariances over the next $N$ time steps are formulated. In Section 3 , under a regularity condition, we prove that multistage look-ahead policies are equivalent to the myopic sensor selection policy that maximizes the trace of the generalized information gain at each time step. In Section 4. the case of uncorrelated measurement noises is considered. The optimal solution is derived analytically for sensor selection when the constraints are temporally separable. When the constraints are temporally inseparable, the sensor selection scheme is obtained by approximately solving an LP. In Section 4, the case of correlated measurement noises is considered. The sensor selection problem is relaxed to a BQP which can be efficiently solved by a Gaussian randomization procedure along with solving an SDP problem. In Section 5, numerical examples are given and discussed. In Section 6, concluding remarks are provided.

\section{Preliminaries}

\subsection{Problem formulation}

We consider a surveillance region of interest (ROI) that is being monitored by a sensor field for potential targets crossing the ROI. The fusion center tracks the target by optimally selecting a fixed number of sensors 
from a large sensor network under some logical or budget constraints. Specifically, we consider a $L$-sensor linear dynamic system

$$
\begin{aligned}
\mathbf{x}_{k+1} & =\mathbf{F}_{k} \mathbf{x}_{k}+\mathbf{w}_{k}, \\
\mathbf{y}_{k}^{i} & =\mathbf{H}_{k}^{i} \mathbf{x}_{k}+\mathbf{v}_{k}^{i}, \quad i=1,2, \ldots, L, \\
\mathbf{z}_{k}^{i} & =\gamma_{k}^{i} \mathbf{H}_{k}^{i} \mathbf{x}_{k}+\gamma_{k}^{i} \mathbf{v}_{k}^{i}, \quad i=1,2, \ldots, L,
\end{aligned}
$$

where $\mathbf{x}_{k} \in \mathbb{R}^{r}, \mathbf{F}_{k} \in \mathbb{R}^{r \times r}$ is an invertible matrix $1 ; \mathbf{y}_{k}^{i} \in \mathbb{R}^{n_{i}}, \mathbf{H}_{k}^{i} \in \mathbb{R}^{n_{i} \times r},\left\{\mathbf{w}_{k}\right\}$ and $\left\{\mathbf{v}_{k}^{i}\right\}$ are both temporally uncorrelated with zero means and invertible covariances $\mathbf{Q}_{k}$ and $\mathbf{R}_{k}^{i}$ respectively. The covariance of the noise $\mathbf{v}_{k} \triangleq\left(\left(\mathbf{v}_{k}^{1}\right)^{\prime}, \ldots,\left(\mathbf{v}_{k}^{L}\right)^{\prime}\right)^{\prime}$ is denoted by $\mathbf{R}_{k} \triangleq \operatorname{Cov}\left(\mathbf{v}_{k}\right)$ which is assumed invertible, $\mathbf{R}_{k}^{i j} \triangleq$ $\operatorname{Cov}\left(\mathbf{v}_{k}^{i}, \mathbf{v}_{k}^{j}\right)$ so that $\mathbf{R}_{k}^{i i}=\mathbf{R}_{k}^{i}$. If the $i$-th sensor is selected, we let $\gamma_{k}^{i}=1$, otherwise $\gamma_{k}^{i}=0$ (see, e.g., [4]); $\gamma_{k} \triangleq\left(\gamma_{k}^{1}, \ldots, \gamma_{k}^{L}\right)^{\prime}$. We shall focus on Equations (1) and (3) for sensor selection. The stacked measurement equation is written as

$$
\mathbf{z}_{k}=\tilde{\mathbf{H}}_{k} \mathbf{x}_{k}+\tilde{\mathbf{v}}_{k},
$$

where

$$
\begin{aligned}
\mathbf{z}_{k} & \triangleq\left(\left(\mathbf{z}_{k}^{1}\right)^{\prime}, \ldots,\left(\mathbf{z}_{k}^{L}\right)^{\prime}\right)^{\prime} \\
\tilde{\mathbf{v}}_{k} & \triangleq\left(\left(\gamma_{k}^{1} \mathbf{v}_{k}^{1}\right)^{\prime}, \ldots,\left(\gamma_{k}^{L} \mathbf{v}_{k}^{L}\right)^{\prime}\right)^{\prime} \\
\tilde{\mathbf{H}}_{k} & \triangleq\left(\left(\gamma_{k}^{1} \mathbf{H}_{k}^{1}\right)^{\prime}, \ldots,\left(\gamma_{k}^{L} \mathbf{H}_{k}^{L}\right)^{\prime}\right)^{\prime}
\end{aligned}
$$

The covariance of the noise $\tilde{\mathbf{v}}_{k}$ is denoted by

$$
\tilde{\mathbf{R}}_{k} \triangleq \operatorname{Cov}\left(\tilde{\mathbf{v}}_{k}\right), \quad \tilde{\mathbf{R}}_{k}^{i j} \triangleq \operatorname{Cov}\left(\gamma_{k}^{i} \mathbf{v}_{k}^{i}, \gamma_{k}^{j} \mathbf{v}_{k}^{j}\right)=\gamma_{k}^{i} \gamma_{k}^{j} \mathbf{R}_{k}^{i j}
$$

Moreover, we denote by $\mathbf{z}_{1: k} \triangleq\left(\mathbf{z}_{1}^{\prime}, \ldots, \mathbf{z}_{k}^{\prime}\right)^{\prime}, \mathbf{x}_{k \mid k} \triangleq \mathbb{E}\left[\mathbf{x}_{k} \mid \mathbf{z}_{1: k}\right], \mathbf{P}_{k \mid k} \triangleq \mathbb{E}\left[\left(\mathbf{x}_{k \mid k}-\mathbf{x}_{k}\right)\left(\mathbf{x}_{k \mid k}-\mathbf{x}_{k}\right)^{\prime}\right]$.

At time $t_{k}$, the fusion center has $\gamma_{k}^{i}, i=1, \ldots, L, \mathbf{x}_{k \mid k}$ and $\mathbf{P}_{k \mid k}$ (or measurements $\mathbf{z}_{1: k}$ ). The fusion center is to design the sensor selection scheme for the next $N$ time steps. At time $t_{k+n}, m_{k+n}$ sensors will be selected from $L$ sensors, for $n=1, \ldots, N$. They will send their measurements, compressed measurements or local estimates to the fusion center. The fusion center makes the final estimates for the state at times $t_{k+n}$, $n=1, \ldots, N$. The problem is how to select $m_{k+n}$ sensors from $L$ sensors (i.e. determine the Boolean decision variables $\left.\gamma_{k+n}^{i}, i=1, \ldots, L\right), n=1, \ldots, N$ that minimize the objective function which is

- either the final estimation error covariance

$$
f_{1} \triangleq \mathbf{P}_{k+N \mid k+N}
$$

\footnotetext{
${ }^{1}$ The invertibility of the transition matrix can be guaranteed in tracking problems, see [21].
} 
- or the average estimation error covariance

$$
f_{2} \triangleq \frac{1}{N} \sum_{n=1}^{N} \mathbf{P}_{k+n \mid k+n} .
$$

The constraint that $m_{k+n}$ sensors are selected from $L$ sensors, $n=1, \ldots, N$ induces a constraint that is temporally separable. Moreover, we shall also consider constraints that are temporally inseparable, for example, energy constraints.

Note that the objective functions (9)-(10) are matrices. Matrix optimization considered here is in the sense that if $x^{*}$ is an optimal solution, then for an arbitrary feasible solution $x, P(x) \succeq P\left(x^{*}\right)$, i.e., $P(x)-$ $P\left(x^{*}\right)$ is a positive semi-definite matrix (see, e.g., [20]).

\subsection{Equivalent Kalman filter for sensor selection}

It is well known that the Kalman filter provides the globally optimal solution if the noises are assumed Gaussian, otherwise it provides the best linear unbiased estimate. It is recursive no matter whether the covariances of noises are invertible or not and is given as follows (see, e.g., [22]),

$$
\begin{aligned}
\mathbf{x}_{k+1 \mid k+1} & =\mathbf{x}_{k+1 \mid k}+\mathbf{K}_{k+1}\left(\mathbf{z}_{k+1}-\tilde{\mathbf{H}}_{k+1} \mathbf{x}_{k+1 \mid k}\right), \\
\mathbf{P}_{k+1 \mid k+1} & =\left(\mathbf{I}-\mathbf{K}_{k+1} \tilde{\mathbf{H}}_{k+1}\right) \mathbf{P}_{k+1 \mid k},
\end{aligned}
$$

where $\mathbf{I}$ is an identity matrix with compatible dimensions,

$$
\begin{aligned}
\mathbf{x}_{k+1 \mid k} & =\mathbf{F}_{k} \mathbf{x}_{k \mid k}, \\
\mathbf{K}_{k+1} & =\mathbf{P}_{k+1 \mid k} \tilde{\mathbf{H}}_{k+1}^{\prime}\left(\tilde{\mathbf{H}}_{k+1} \mathbf{P}_{k+1 \mid k} \tilde{\mathbf{H}}_{k+1}^{\prime}+\tilde{\mathbf{R}}_{k+1}\right)^{+}, \\
\mathbf{P}_{k+1 \mid k} & =\mathbf{F}_{k} \mathbf{P}_{k \mid k} \mathbf{F}_{k}^{\prime}+\mathbf{Q}_{k} .
\end{aligned}
$$

The superscript “+” means Moore-Penrose generalized inverse (see, e.g., [23]) 2. If $\mathbf{P}_{k+1 \mid k}, \mathbf{P}_{k+1 \mid k+1}$ and $\tilde{\mathbf{R}}_{k+1}$ are invertible (for example, the case that all $L$ sensors are selected), then we have the following equivalent Kalman filter

$$
\begin{aligned}
\mathbf{x}_{k+1 \mid k+1} & =\mathbf{P}_{k+1 \mid k+1}\left(\mathbf{P}_{k+1 \mid k}^{-1} \mathbf{x}_{k+1 \mid k}+\tilde{\mathbf{H}}_{k+1}^{\prime} \tilde{\mathbf{R}}_{k+1}^{-1} \mathbf{z}_{k+1}\right), \\
\mathbf{P}_{k+1 \mid k+1} & =\left(\mathbf{P}_{k+1 \mid k}^{-1}+\tilde{\mathbf{H}}_{k+1}^{\prime} \tilde{\mathbf{R}}_{k+1}^{-1} \tilde{\mathbf{H}}_{k+1}\right)^{-1},
\end{aligned}
$$

which is usually called the information filter and $\tilde{\mathbf{H}}_{k+1}^{\prime} \tilde{\mathbf{R}}_{k+1}^{-1} \tilde{\mathbf{H}}_{k+1}$ is called the information gain (see e.g., [24]). Once the sensors are selected, the covariance of the noise vector of the selected sensors is invertible.

\footnotetext{
${ }^{2}$ Here, the Moore-Penrose generalized inverse is used since the $\left(\tilde{\mathbf{H}}_{k+1} \mathbf{P}_{k+1 \mid k} \tilde{\mathbf{H}}_{k+1}^{\prime}+\tilde{\mathbf{R}}_{k+1}\right)$ may not be invertible. The reason is that $\tilde{\mathbf{H}}_{k+1}$ and $\tilde{\mathbf{R}}_{k+1}$ defined by (7) and (8) for the sensor selection problem include the decision variables $\left\{\gamma_{k+1}^{1}, \ldots, \gamma_{k+1}^{L}\right\}$ which have $L-m_{k+1}$ number of zeros.
} 
Note that we assumed that $\mathbf{Q}_{k}, k=1,2, \ldots$, are invertible, and it is easy to check that $\mathbf{P}_{k+1 \mid k}$ and $\mathbf{P}_{k+1 \mid k+1}$ which are updated by the Kalman Filter based on the selected sensors are also invertible. Here, however, $\tilde{\mathbf{R}}_{k+1}$ are not invertible, since there are $L-m_{k+1}$ number of zeros in the decision variables $\left\{\gamma_{k+1}^{1}, \ldots, \gamma_{k+1}^{L}\right\}$. Thus, we first prove that, for the dynamic system (1) and (4) defined under sensor selection where $\tilde{\mathbf{R}}_{k+1}$ are not invertible, there still exists an equivalent Kalman filter similar to (16)-(17).

Theorem 2.1. For the dynamic system defined by (1) and (4) under sensor selection, we have the following equivalent Kalman filter (generalized information filter)

$$
\begin{aligned}
\mathbf{x}_{k+1 \mid k+1} & =\mathbf{P}_{k+1 \mid k+1}\left(\mathbf{P}_{k+1 \mid k}^{-1} \mathbf{x}_{k+1 \mid k}+\tilde{\mathbf{H}}_{k+1}^{\prime} \tilde{\mathbf{R}}_{k+1}^{+} \mathbf{z}_{k+1}\right) \\
\mathbf{P}_{k+1 \mid k+1} & =\left(\mathbf{P}_{k+1 \mid k}^{-1}+\tilde{\mathbf{H}}_{k+1}^{\prime} \tilde{\mathbf{R}}_{k+1}^{+} \tilde{\mathbf{H}}_{k+1}\right)^{-1}
\end{aligned}
$$

Proof. See appendix.

The key difference in Theorem 2.1 is that $\tilde{\mathbf{R}}_{k+1}^{-1}$ where $\tilde{\mathbf{R}}_{k+1}$ is invertible in (16)-(17) has been replaced by $\tilde{\mathbf{R}}_{k+1}^{+}$in (18)-19]. Due to this difference, $\tilde{\mathbf{H}}_{k+1}^{\prime} \tilde{\mathbf{R}}_{k+1}^{+} \tilde{\mathbf{H}}_{k+1}$ will be called generalized information gain. Notice that $\mathbf{P}_{k+1 \mid k+1}$ is a function of $\gamma_{k+1}^{1}, \ldots, \gamma_{k+1}^{L}$, since $\tilde{\mathbf{H}}_{k+1}$ and $\tilde{\mathbf{R}}_{k+1}$ are functions of $\gamma_{k+1}^{1}, \ldots, \gamma_{k+1}^{L}$. Thus, it is denoted by $\mathbf{P}_{k+1 \mid k+1}\left(\gamma_{k+1}^{1}, \ldots, \gamma_{k+1}^{L}\right)$. Similarly, $\mathbf{P}_{k+n \mid k+n}\left(\gamma_{k+1}^{1}, \ldots, \gamma_{k+1}^{L}, \ldots, \gamma_{k+n}^{1}, \ldots, \gamma_{k+n}^{L}\right)$ is a function of $\gamma_{k+1}^{1}, \ldots, \gamma_{k+1}^{L}, \ldots, \gamma_{k+n}^{1}, \ldots, \gamma_{k+n}^{L}$, for $n=1, \ldots, N$.

It is the generalized information filter based on Moore-Penrose generalized inverse that helps us decouple the multistage look-ahead policies to an equivalent myopic sensor selection policy that maximizes the generalized information gain with a lower computational complexity in Section 3 . Another advantage is that the sensor selection problem formulated by the use of the Moore-Penrose generalized inverse only relies on Boolean decision variables without introducing additional decision variables and can efficiently take advantage of the structure of the measurement noise to obtain the optimal solution and efficient algorithms.

\subsection{Optimization problems for sensor selection}

Thus, by using Theorem 2.1, the two sensor selection problems can be stated as

$$
\begin{aligned}
\min _{\gamma_{k+n}^{i}} & \mathbf{P}_{k+N \mid k+N}\left(\gamma_{k+1}^{1}, \ldots, \gamma_{k+1}^{L}, \ldots, \gamma_{k+N}^{1}, \ldots, \gamma_{k+N}^{L}\right) \\
& =\left(\mathbf{P}_{k+N \mid k+N-1}^{-1}+\tilde{\mathbf{H}}_{k+N}^{\prime} \tilde{\mathbf{R}}_{k+N}^{+} \tilde{\mathbf{H}}_{k+N}\right)^{-1} \\
\text { subject to } \quad & \sum_{i=1}^{L} \gamma_{k+n}^{i}=m_{k+n}, n=1, \ldots, N, \\
& \gamma_{k+n}^{i} \in\{0,1\}, i=1,2, \ldots, L, n=1, \ldots, N,
\end{aligned}
$$


and

$$
\begin{aligned}
\min _{\gamma_{k+n}^{i}} & \sum_{n=1}^{N} \mathbf{P}_{k+n \mid k+n}\left(\gamma_{k+1}^{1}, \ldots, \gamma_{k+1}^{L}, \ldots, \gamma_{k+N}^{1}, \ldots, \gamma_{k+N}^{L}\right) \\
& =\sum_{n=1}^{N}\left(\mathbf{P}_{k+n \mid k+n-1}^{-1}+\tilde{\mathbf{H}}_{k+n}^{\prime} \tilde{\mathbf{R}}_{k+n}^{+} \tilde{\mathbf{H}}_{k+n}\right)^{-1} \\
\text { subject to } \quad & \sum_{i=1}^{L} \gamma_{k+n}^{i}=m_{k+n}, n=1, \ldots, N, \\
& \gamma_{k+n}^{i} \in\{0,1\}, i=1,2, \ldots, L, n=1, \ldots, N .
\end{aligned}
$$

\section{Generalized Information Measure for Sensor Selection}

In this section, we consider some properties of the optimization problems presented in Section 2.3 that will simplify their solution. We will show that if the primal sensor selection problem (20) has an optimal solution, then both the problem (20) and the problem (23) can be transformed to equivalent optimization problems that maximize an information measure at each time step.

Lemma 3.1. Consider two optimization problems:

$$
\begin{array}{lll}
\left(A_{1}\right) & \max _{\mathbf{x} \in \mathcal{S}} & \mathbf{M}(\mathbf{x}), \\
\left(A_{2}\right) & \max _{\mathbf{x} \in \mathcal{S}} & \operatorname{tr}(\mathbf{M}(\mathbf{x})),
\end{array}
$$

where $\mathbf{M}(\mathbf{x})$ is a matrix for an arbitrary $\mathbf{x} \in \mathcal{S} ; \mathcal{S}$ specifies the constraint on the decision variable $\mathbf{x}$. If the problem $\left(A_{1}\right)$ has an optimal solution, then the problem $\left(A_{1}\right)$ is equivalent to $\left(A_{2}\right)$.

Proof. See appendix.

Lemma 3.2. Consider two optimization problems:

$$
\begin{aligned}
& \left(B_{1}\right) \quad \min _{\mathbf{x}_{i} \in \mathcal{S}_{i}, i=1, \ldots, n} \mathbf{M}_{n}\left(\mathbf{x}_{1}, \ldots, \mathbf{x}_{n}\right), \text { for } n=1, \ldots, N, \\
& \left(B_{2}\right) \quad \min _{\mathbf{x}_{n} \in \mathcal{S}_{n}, n=1, \ldots, N} \sum_{n=1}^{N} \mathbf{M}_{n}\left(\mathbf{x}_{1}, \ldots, \mathbf{x}_{n}\right)
\end{aligned}
$$

where $\mathbf{M}_{n}\left(\mathbf{x}_{1}, \ldots, \mathbf{x}_{n}\right)$ is a function of decision variables $\mathbf{x}_{1}, \ldots, \mathbf{x}_{n}$, for $n=1, \ldots, N$. If the optimal solution that minimizes $\mathbf{M}_{n}\left(\mathbf{x}_{1}, \ldots, \mathbf{x}_{n}\right),\left(\mathbf{x}_{1}^{*}, \ldots, \mathbf{x}_{n}^{*}\right)$, is the same as the one that minimizes $\mathbf{M}_{n+1}\left(\mathbf{x}_{1}, \ldots, \mathbf{x}_{n+1}\right)$, for $n=1, \ldots, N-1$, then the optimal solution that minimizes $\mathbf{M}_{N}\left(\mathbf{x}_{1}, \ldots, \mathbf{x}_{N}\right)\left(\left(B_{1}\right)\right.$ with $\left.n=N\right)$ is the same as that for $\left(B_{2}\right)$. 
Proof. See appendix.

Based on Lemma 3.2, the solution to both the problem (20) and the problem (23) can be simplified and obtained by solving $N$ optimization problems separately.

Lemma 3.3. If the primal sensor selection problem (20) has an optimal solution, then both the problem (20) and the problem (23) can be transformed to the equivalent problem that solves $N$ optimization problems that maximize $\tilde{\mathbf{H}}_{k+n}^{\prime} \tilde{\mathbf{R}}_{k+n}^{+} \tilde{\mathbf{H}}_{k+n}, n=1, \ldots, N$ respectively, i.e.,

$$
\begin{aligned}
\max _{\gamma_{k+n}^{i}} & \tilde{\mathbf{H}}_{k+n}^{\prime} \tilde{\mathbf{R}}_{k+n}^{+} \tilde{\mathbf{H}}_{k+n} \quad \text { for } n=1, \ldots, N, \\
\text { subject to } & \sum_{i=1}^{L} \gamma_{k+n}^{i}=m_{k+n}, \\
& \gamma_{k+n}^{i} \in\{0,1\}, i=1,2, \ldots, L,
\end{aligned}
$$

where $\tilde{\mathbf{H}}_{k+n}$ and $\tilde{\mathbf{R}}_{k+n}$ are defined in Equations (7) and (8) respectively; the superscript " " indicates Moore-Penrose generalized inverse [23]. That is, the problems (20), (23) and (28) have the same optimal solution.

Proof. See appendix.

Remark 3.4. Lemma 3.3 shows that multistage look-ahead policies, i.e., the problem (20) and the problem (23), are equivalent to a myopic sensor selection policy that maximizes the generalized information gain with a lower computational complexity. Why do the problem (20) and the problem (23) with different objectives have the same optimal solution? The main reason is that the objectives and constraints are temporally separable. For example, consider $m_{k+n}=1$, i.e., select one sensor at each time step, if there is a sensor which has the smallest noise and provides the most information at each time step, then the selection of the sensor at each time step is the optimal sensor selection scheme no matter whether the objective is the final estimation error covariance or the average estimation error covariance.

Moreover, based on Lemmas 3.1 and 3.3, we have the following theorem.

Theorem 3.5. If the primal sensor selection problem (20) has an optimal solution, both the problem (20) and the problem (23) can be transformed to the equivalent problem requiring the solution of $N$ optimization problems that maximize $\operatorname{tr}\left(\tilde{\mathbf{H}}_{k+n}^{\prime} \tilde{\mathbf{R}}_{k+n}^{+} \tilde{\mathbf{H}}_{k+n}\right), n=1, \ldots, N$ respectively, i.e.,

$$
\begin{aligned}
\max _{\gamma_{k+n}^{i}} & \operatorname{tr}\left(\tilde{\mathbf{H}}_{k+n}^{\prime} \tilde{\mathbf{R}}_{k+n}^{+} \tilde{\mathbf{H}}_{k+n}\right) \quad \text { for } n=1, \ldots, N, \\
\text { subject to } & \sum_{i=1}^{L} \gamma_{k+n}^{i}=m_{k+n}, \\
& \gamma_{k+n}^{i} \in\{0,1\}, i=1,2, \ldots, L,
\end{aligned}
$$


where $\tilde{\mathbf{H}}_{k+n}$ and $\tilde{\mathbf{R}}_{k+n}$ are defined in Equations (7) and (8) respectively. That is, the problems (20), (23) and (29) have the same optimal solution.

Remark 3.6. Theorem 3.5 shows that both the minimization of the final estimation error covariance and minimization of the average estimation error covariance are equivalent to maximization of the trace of the generalized information gain of each time step. Thus, the objective function

$$
\operatorname{tr}\left(\tilde{\mathbf{H}}_{k+n}^{\prime} \tilde{\mathbf{R}}_{k+n}^{+} \tilde{\mathbf{H}}_{k+n}\right)
$$

of the problem (28), i.e., trace of the generalized information gain, is defined as a measure of information that the selected sensors provide at $(k+n)$-th time step. Determinant of the generalized information gain can be similarly defined as a measure of information if $\tilde{\mathbf{H}}_{k+n}^{\prime} \tilde{\mathbf{R}}_{k+n}^{+} \tilde{\mathbf{H}}_{k+n}$ is a positive definite matrix. When the measurement noises are assumed independent, more information measures based on information gain can be formulated, see e.g., [19].

Furthermore, the information measure (30) has an advantage that it does not depend on pdfs of the states and measurements, but only relies on covariances of noises and the measurement matrices. Maximizing this measure can be employed as an alternative criterion for sensor selection, which will be used in sensor selection problems when the constraints are temporally inseparable in the following sections. When pdfs are known, it is better to try to use the information criteria based on pdfs such as optimization of Fisher information, entropy or mutual information for sensor selection (see, e.g., [2]).

\section{Sensor Selection Schemes for Uncorrelated Sensor Measurement Noises}

\subsection{Optimal Sensor Selection Scheme for Temporally Separable Constraints}

When sensor measurement noises are uncorrelated and the constraints are temporally separable, we have the following result that defines the optimal sensor selection scheme.

Theorem 4.1. Let the information measure corresponding to the $i$-th sensor at $(k+n)$-th time be denoted as $a_{k+n}^{i} \triangleq \operatorname{tr}\left(\left(\mathbf{H}_{k+n}^{i}\right)^{\prime}\left(\mathbf{R}_{k+n}^{i}\right)^{-1} \mathbf{H}_{k+n}^{i}\right), i=1, \ldots$, L. Let $\left\{a_{k+n}^{i_{1}}, \ldots, a_{k+n}^{i_{L}}\right\}$ denote $\left\{a_{k+n}^{1}, \ldots\right.$, $\left.a_{k+n}^{L}\right\}$ rearranged in descending order. If the problem (20) has an optimal solution, then the optimal sensor selection scheme for both the problem (20) and the problem (23) is $\gamma_{k+n}^{i_{1}}=1, \ldots, \gamma_{k+n}^{i_{m_{k+n}}}=1$, $\gamma_{k+n}^{i_{m_{k+n+1}}}=0, \ldots, \gamma_{k+n}^{i_{L}}=0$, for $n=1, \ldots, N$. The optimality of sensor selection scheme is in the sense of either the minimization of the covariance of the final estimation error (9) or the average estimation error (10) or maximization of the information measure (30). If the problem (20) does not have an optimal solution, the optimality of the sensor selection scheme is only in the sense of maximization of the information measure (30).

Proof. If the measurement noises are uncorrelated between sensors, then $\tilde{\mathbf{R}}_{k+n}$ is a block diagonal matrix 
with $\tilde{\mathbf{R}}_{k+n}^{i j}=0, i \neq j$. Thus,

$$
\tilde{\mathbf{R}}_{k+n}^{+}=\left(\operatorname{diag}\left(\gamma_{k+n}^{1} R_{k+n}^{1}, \ldots, \gamma_{k+n}^{L} R_{k+n}^{L}\right)\right)^{+}=\operatorname{diag}\left(\gamma_{k+n}^{1}\left(R_{k+n}^{1}\right)^{-1}, \ldots, \gamma_{k+n}^{L}\left(R_{k+n}^{L}\right)^{-1}\right)
$$

which follows from the definition of Moore-Penrose generalized inverse. Moreover, by Theorem 3.5, the problem (20) is equivalent to

$$
\begin{array}{cl}
\max _{\gamma_{k+n}^{i}} & \operatorname{tr}\left(\tilde{\mathbf{H}}_{k+n}^{\prime} \tilde{\mathbf{R}}_{k+n}^{+} \tilde{\mathbf{H}}_{k+n}\right) \quad \text { for } n=1, \ldots, N, \\
& =\sum_{i=1}^{L} \gamma_{k+n}^{i} \operatorname{tr}\left(\left(\mathbf{H}_{k+n}^{i}\right)^{\prime}\left(\mathbf{R}_{k+n}^{i}\right)^{-1} \mathbf{H}_{k+n}^{i}\right) \\
\text { subject to } \quad & \sum_{i=1}^{L} \gamma_{k+n}^{i}=m_{k+n}, \\
& \gamma_{k+n}^{i} \in\{0,1\}, i=1,2, \ldots, L .
\end{array}
$$

If we define $a_{k+n}^{i} \triangleq \operatorname{tr}\left(\left(\mathbf{H}_{k+n}^{i}\right)^{\prime}\left(\mathbf{R}_{k+n}^{i}\right)^{-1} \mathbf{H}_{k+n}^{i}\right), i=1, \ldots, L$ and $\left\{a_{k+n}^{i_{1}}, \ldots, a_{k+n}^{i_{L}}\right\}$ denotes $\left\{a_{k+n}^{1}, \ldots\right.$, $\left.a_{k+n}^{L}\right\}$ rearranged in descending order, then the optimal solution is $\gamma_{k+n}^{i_{1}}=1, \ldots, \gamma_{k+n}^{i_{m_{k+n}}}=1, \gamma_{k+n}^{i_{m_{k+n+1}}}=$ $0, \ldots, \gamma_{k+n}^{i_{L}}=0$.

\subsection{Extension to Temporally Inseparable Constraints}

Many constraints on sensor selection can be represented as linear equalities or inequalities such as logical constraints and budget constraints (see, e.g., [3, 4]). Let us denote linear equalities or inequalities as follows

$$
\mathbf{a}_{p}^{\prime} \gamma \unrhd b_{p}, p=1, \ldots, P
$$

where

$$
\gamma \triangleq\left(\gamma_{k+1}^{\prime}, \ldots, \gamma_{k+N}^{\prime}\right)^{\prime}, \quad \gamma_{k+n} \triangleq\left(\gamma_{k+n}^{1}, \ldots, \gamma_{k+n}^{L}\right)^{\prime}, \text { for } n=1, \ldots, N
$$

$\mathbf{a}_{p}$ is a vector with a compatible dimension and $b_{p}$ is a scalar; " $\unrhd$ " can represent either " $\geq$ " " $\leq$ " or "=" for each $n$. In general, these constraints are temporally inseparable, which makes the optimization problems with objectives (20) and (23) not separable and highly nonlinear in variables $\gamma_{k+n}^{i}$. The corresponding optimization problems are very hard to solve.

However, from Remark 3.6 the trace of generalized information, $\operatorname{tr}\left(\tilde{\mathbf{H}}_{k+n}^{\prime} \tilde{\mathbf{R}}_{k+n}^{+} \tilde{\mathbf{H}}_{k+n}\right)$, can be defined as the measure of information that selected sensors provide. Thus, we can try to maximize the available information gain from time $k+1$ to $k+N$ by optimizing the selection of sensors so that better estimation 
performance can be expected. We shall consider the following objective (i.e, sum of the weighted information measure)

$$
f_{3} \triangleq \sum_{n=1}^{N} \omega_{n} \operatorname{tr}\left(\tilde{\mathbf{H}}_{k+n}^{\prime} \tilde{\mathbf{R}}_{k+n}^{+} \tilde{\mathbf{H}}_{k+n}\right),
$$

where $\omega_{n}, n=1, \ldots, N$ are weights which place different importance on different time steps. For example, if the state estimation at the final time is more important, a larger weight $\omega_{N}$ can be used. If the state estimation of each time step is equally important, an equal weight structure $\omega_{1}=\cdots=\omega_{N}$ can be used. Therefore, we consider the following optimization problem for sensor selection:

$$
\begin{aligned}
\max _{\gamma_{k+n}^{i}} & \sum_{n=1}^{N} \omega_{n} \operatorname{tr}\left(\tilde{\mathbf{H}}_{k+n}^{\prime} \tilde{\mathbf{R}}_{k+n}^{+} \tilde{\mathbf{H}}_{k+n}\right) \\
\text { subject to } & \mathbf{a}_{p}^{\prime} \gamma \unrhd b_{p}, p=1, \ldots, P, \\
& \gamma_{k+n}^{i} \in\{0,1\}, i=1,2, \ldots, L, n=1, \ldots, N .
\end{aligned}
$$

Since sensor measurement noises are assumed uncorrelated in this section, by Equation (31), the problem (35) is equivalent to

$$
\begin{aligned}
\max _{\gamma_{k+n}^{i}} & \sum_{n=1}^{N} \omega_{n} \sum_{i=1}^{L} \gamma_{k+n}^{i} \operatorname{tr}\left(\left(\mathbf{H}_{k+n}^{i}\right)^{\prime}\left(\mathbf{R}_{k+n}^{i}\right)^{-1} \mathbf{H}_{k+n}^{i}\right) \\
\text { subject to } & \mathbf{a}_{p}^{\prime} \gamma \unrhd b_{p}, p=1, \ldots, P, \\
& \gamma_{k+n}^{i} \in\{0,1\}, i=1,2, \ldots, L, n=1, \ldots, N,
\end{aligned}
$$

which is a Boolean linear programming (BLP) problem and the optimal objective function value is denoted by $f_{B L P}^{*}$. It can be relaxed by replacing the nonconvex constraints $\gamma_{k+n}^{i} \in\{0,1\}$ with the convex constraints $0 \leq \gamma_{k+n}^{i} \leq 1, i=1,2, \ldots, L, n=1, \ldots, N$. Thus, we have the following LP problem:

$$
\begin{aligned}
\max _{\gamma_{k+n}^{i}} & \operatorname{tr}\left(\Gamma \mathbf{D}^{\prime}\right) \\
\text { subject to } & \mathbf{a}_{p}^{\prime} \gamma \unrhd b_{p}, p=1, \ldots, P, \\
& 0 \leq \gamma_{k+n}^{i} \leq 1, i=1,2, \ldots, L, n=1, \ldots, N,
\end{aligned}
$$

where $\gamma$ is defined by (33); $\Gamma$ is a $L \times N$ matrix with $i$-th row and $n$-th column element being $\gamma_{k+n}^{i}$, i.e,

$$
\Gamma \triangleq\left(\begin{array}{cccc}
\gamma_{k+1}^{1} & \gamma_{k+2}^{1} & \cdots & \gamma_{k+N}^{1} \\
\gamma_{k+1}^{2} & \gamma_{k+2}^{2} & \cdots & \gamma_{k+N}^{2} \\
\vdots & \vdots & \ddots & \vdots \\
\gamma_{k+1}^{L} & \gamma_{k+2}^{L} & \cdots & \gamma_{k+N}^{L}
\end{array}\right)
$$

and $\mathbf{D}$ is a $L \times N$ matrix

$$
\mathbf{D} \triangleq\left(d_{i n}\right)_{L \times N}, \quad d_{i n} \triangleq \omega_{n} \operatorname{tr}\left(\left(\mathbf{H}_{k+n}^{i}\right)^{\prime}\left(\mathbf{R}_{k+n}^{i}\right)^{-1} \mathbf{H}_{k+n}^{i}\right) .
$$


It is well known that LP problems can be solved efficiently. The solution of the problem (37) is denoted by $\left(\gamma_{k+n}^{i}\right)_{L P}^{*}, i=1, \ldots, L, n=1, \ldots, N$. The corresponding objective function is denoted by $f_{L P}^{*}$. Note that the feasible solution set of the problem (37) contains that of the problem (36) so that $f_{B L P}^{*} \leq f_{L P}^{*}$. Based on $\left(\gamma_{k+n}^{i}\right)_{L P}^{*}$, we can generate a suboptimal feasible solution of the problem (36) denoted by $\hat{\gamma}_{k+n}^{i}$, $i=1, \ldots, L, n=1, \ldots, N$. The corresponding objective function is denoted by $\hat{f}_{B L P}$ and $\hat{f}_{B L P} \leq f_{B L P}^{*}$. The difference $g=f_{L P}^{*}-\hat{f}_{B L P}$ is called the gap in [3]. The gap is useful in evaluating the performance of the suboptimal solution $\hat{\gamma}_{k+n}^{i}$. We can say $\hat{\gamma}_{k+n}^{i}$ is no more than $g$-suboptimal.

Note that the procedure of generating a feasible solution of the problem (36) from $\left(\gamma_{k+n}^{i}\right)_{L P}^{*}$ is problem dependent, i.e., relying on the equalities or inequalities (32) and the Boolean constraint. As an illustration, let us consider a representative example. Besides the temporally separable constraints (21) and (22), we consider an energy constraint which is temporally inseparable as follows

$$
\sum_{n=1}^{N} \gamma_{k+n}^{i} \leq m_{k}^{i}, i=1, \ldots, L
$$

which means that the $i$-th sensor can only be selected $m_{k}^{i}$ times from time $k+1$ to time $k+N\left(m_{k}^{i}<N\right)$, for $i=1, \ldots, L$. Thus, the specific form of the optimization problem (37) with the constraints (21) and (40) can be represented to

$$
\begin{aligned}
\max _{\gamma_{k+n}^{i}} & \operatorname{tr}\left(\Gamma \mathbf{D}^{\prime}\right) \\
\text { subject to } & \mathbf{a}_{p}^{\prime} \gamma \unrhd b_{p}, p=1, \ldots, P, \\
& 0 \leq \gamma_{k+n}^{i} \leq 1, i=1,2, \ldots, L, n=1, \ldots, N,
\end{aligned}
$$

where $\Gamma$ and $\gamma$ are defined by (38) and (33) respectively; $P=N+L$,

$$
\begin{aligned}
\mathbf{a}_{p} \triangleq & \left(\mathbf{c}_{1, p}^{\prime}, \ldots, \mathbf{c}_{N, p}^{\prime}\right)^{\prime}, \mathbf{c}_{n, p} \triangleq\left\{\begin{array}{l}
\mathbf{1}, \quad p=n, \\
\mathbf{0}, \quad p \neq n,
\end{array} \quad n=1 \ldots, N,\right. \\
b_{p} \triangleq & m_{k+p}, " \unrhd " \text { means " }=", \\
& \quad \text { for } p=1, \ldots, N,(\text { corresponds to the constraints (21) }) ; \\
\mathbf{a}_{p} \triangleq & \left(\mathbf{1}_{i}^{\prime}, \ldots, \mathbf{1}_{i}^{\prime}\right)^{\prime}, \\
b_{p} \triangleq & m_{k}^{i}, " \unrhd " \text { means " } \leq ", \\
& \quad \text { for } p=N+i, i=1, \ldots, L,(\text { corresponds to the constraints (40) }),
\end{aligned}
$$

where 1 and $\mathbf{0}$ denote $L$-dimensional vectors with 1 entries and 0 entries respectively and $\mathbf{1}_{i}$ means an $L$ dimensional vector whose $i$-th entry is 1 others are 0 s.

The sensor selection scheme with the energy constraint for uncorrelated sensors is described by the following algorithm. 
Algorithm 4.2 (Sensor selection scheme with the energy constraint for uncorrelated sensors).

- Step 1: Given an optimal solution of (41) $\left(\gamma_{k+n}^{i}\right)_{L P}^{*}$, obtain the optimal objective function $f_{L P}^{*}$.

- Step 2: Generate a feasible solution of the problem (36) with the constraints (21), (22) and (40) from $\left(\gamma_{k+n}^{i}\right)_{L P}^{*}$ as follows.

We generate the feasible solution based on the importance (weight) of the information of each time step. Without loss of generality, assume that $\omega_{1} \leq \omega_{2} \leq \ldots \leq \omega_{N}$. Thus, we generate the selection scheme from the $N$-th time step to the first time step. Set the index set of candidate sensors $\dot{\mathrm{i}} \triangleq\{1, \ldots, L\}$.

- Iteratively generate $\hat{\gamma}_{k+N-n}^{i}$ for the $(N-n)$-th time step, $n=0, \ldots,(N-1)$ as follows: for $n=0:(N-1)$

$$
\hat{\gamma}_{k+N-n}^{i} \triangleq\left\{\begin{array}{ll}
1, & \text { if } i \in \dot{\mathrm{i}}_{1}, \\
0, & \text { if } i \in \dot{\mathrm{i}}_{2},
\end{array}, \text { for } i=1, \ldots, L,\right.
$$

where $\dot{\mathrm{n}}_{1}$ is the index set of the first $m_{k+N-n}$ maximum entries of $\left(\left(\gamma_{k+N-n}^{1}\right)_{L P}^{*}, \ldots,\left(\gamma_{k+N-n}^{L}\right)_{L P}^{*}\right)$ in the index set of candidate sensors $\dot{\mathbf{i}}$ and denote $\dot{\mathrm{i}}_{2}=\dot{\mathrm{i}}-\dot{\mathrm{i}}_{1}$. Set $m_{k}^{i}:=m_{k}^{i}-1$, for $i \in \dot{\mathrm{i}}_{1}$. Update the index set of candidate sensors $\dot{\mathrm{i}} \triangleq\left\{i: m_{k}^{i}>0, i=1, \ldots, L\right\}$.

end

- Step 3: Output g-suboptimal solution $\hat{\gamma}_{k+n}^{i}, i=1, \ldots, L, n=1, \ldots, N$ and the corresponding objective $\hat{f}_{B L P}$, where $g=f_{L P}^{*}-\hat{f}_{B L P}$ is the gap.

Here, to construct the feasible solution satisfying the constraints (21), (22) and (40), we employ the equation (44). The main computation complexity is in Step 1 where an LP problem needs to be solved. Illustrative examples will be presented in Section 6

\section{Sensor Selection Schemes for Correlated Sensor Measurement Noises}

In this section, for correlated sensor measurement noises, we again determine the sensor selection scheme by maximizing the weighted information measure:

$$
\begin{aligned}
\max _{\gamma_{k+n}^{i}} & \sum_{n=1}^{N} \omega_{n} \operatorname{tr}\left(\tilde{\mathbf{H}}_{k+n}^{\prime} \tilde{\mathbf{R}}_{k+n}^{+} \tilde{\mathbf{H}}_{k+n}\right) \\
\text { subject to } & \mathbf{a}_{p}^{\prime} \gamma \unrhd b_{p}, p=1, \ldots, P, \\
& \gamma_{k+n}^{i} \in\{0,1\}, i=1,2, \ldots, L .
\end{aligned}
$$

where the linear constraints are defined in (32) that may include both the temporally separable and inseparable constraints. Since sensor measurement noises are correlated, to obtain the optimal solution, an exhaustive 
search is necessary since $\tilde{\mathbf{R}}_{k+n}$ has no special structure such as it being a diagonal matrix. For the simplest case of the temporally separable constraint (21) and $N=1$, there are a total of $\left(\begin{array}{c}L \\ m_{k+n}\end{array}\right)$ feasible solutions. For large $L$ and $m_{k+n}$, such an exhaustive search may not be feasible in real time. Thus, to make the solution computationally more efficient, the problem (45) is approximately solved by replacing $\tilde{\mathbf{R}}_{k+n}^{+}$by $\mathbf{R}_{k+n}^{-1}$. This approximation is lossless for the case of uncorrelated sensor noises and temporally separable constraint (i.e., does not change the optimal solution in Theorem 4.1). More discussion on approximation loss for different dependences will be given in Section 6, Thus, we consider the approximate problem

$$
\begin{aligned}
\max _{\gamma_{k+n}^{i}} & \sum_{n=1}^{N} \omega_{n} \operatorname{tr}\left(\tilde{\mathbf{H}}_{k+n}^{\prime} \mathbf{R}_{k+n}^{-1} \tilde{\mathbf{H}}_{k+n}\right) \\
\text { subject to } & \mathbf{a}_{p}^{\prime} \gamma \unrhd b_{p}, p=1, \ldots, P, \\
& \gamma_{k+n}^{i} \in\{0,1\}, i=1,2, \ldots, L .
\end{aligned}
$$

Moreover, from the definition of $\tilde{\mathbf{H}}_{k+n}(7)$, we have

$$
\begin{aligned}
& \operatorname{tr}\left(\tilde{\mathbf{H}}_{k+n}^{\prime} \mathbf{R}_{k+n}^{-1} \tilde{\mathbf{H}}_{k+n}\right) \\
= & \operatorname{tr}\left(\sum_{i=1}^{L} \sum_{s=1}^{L} \gamma_{k+n}^{i} \gamma_{k+n}^{s}\left(\mathbf{H}_{k+n}^{i}\right)^{\prime} \mathbf{T}_{k+n}^{i s} \mathbf{H}_{k+n}^{s}\right) \\
= & \sum_{i=1}^{L} \sum_{s=1}^{L} \gamma_{k+n}^{i} \gamma_{k+n}^{s} \operatorname{tr}\left(\left(\mathbf{H}_{k+n}^{i}\right)^{\prime} \mathbf{T}_{k+n}^{i s} \mathbf{H}_{k+n}^{s}\right) \\
= & -\gamma_{k+n}^{\prime} \mathbf{B}_{k+n} \gamma_{k+n},
\end{aligned}
$$

where $\mathbf{T}_{k+n}^{i s}$ is the $i$-th row block and $s$-th column block of the matrix $\mathbf{T}_{k+n}, \mathbf{T}_{k+n} \triangleq \mathbf{R}_{k+n}^{-1}$; the $i$-th row and $s$-th column of $\mathbf{B}_{k+n}$ is $-\operatorname{tr}\left(\left(\mathbf{H}_{k+n}^{i}\right)^{\prime} \mathbf{T}_{k+n}^{i s} \mathbf{H}_{k+n}^{s}\right)$. Thus, the problem is equivalent to solving the following Boolean quadratic programming $(\mathrm{BQP})$ problem

$$
\begin{aligned}
\min _{\gamma_{k+n}^{i}} & \sum_{n=1}^{N} \omega_{n} \gamma_{k+n}^{\prime} \mathbf{B}_{k+n} \gamma_{k+n} \\
\text { subject to } & \mathbf{a}_{p}^{\prime} \gamma \unrhd b_{p}, p=1, \ldots, P, \\
& \gamma_{k+n}^{i} \in\{0,1\}, i=1,2, \ldots, L, n=1, \ldots, N .
\end{aligned}
$$

For this problem, however, it is still hard to obtain an optimal solution, since the nonconvex Boolean constraints and $\mathbf{B}_{k+n}$ may not be a positive semi-definite matrix. It is known to belong to the class of NP-hard problems. Fortunately, this class of problems can be solved by a recently developed computationally efficient approximation technique (see, e.g., [25]). We apply it to the problem (48) as follows. 
By semidefinite relaxation (SDR) technique (see, e.g., [20, 25]), the problem (48) can be relaxed to

$$
\begin{array}{cl}
\min _{\mathbf{X} \in \mathbb{S}^{(N L+1)}, \mathbf{X} \succeq \mathbf{0}} & \operatorname{tr}(\mathbf{C X}) \\
\text { subject to } & \operatorname{tr}\left(\mathbf{E}_{p} \mathbf{X}\right) \unrhd\left(4 b_{p}-\mathbf{1}^{\prime} \operatorname{diag}\left(\mathbf{a}_{p}\right) \mathbf{1}\right), \quad p=1, \ldots, P, \\
& \operatorname{tr}\left(\mathbf{F}_{s} \mathbf{X}\right)=1, s=1, \ldots,(N L+1),
\end{array}
$$

where $\mathbf{F}_{s}$ is a matrix with $s$-row and $s$-column $\mathbf{F}_{s}(s, s)=1$, others are equal 0 , for $s=1, \ldots,(N L+1)$,

$$
\begin{aligned}
\mathbf{E}_{p} & \triangleq\left(\begin{array}{cc}
\operatorname{diag}\left(\mathbf{a}_{p}\right) & \mathbf{1} \\
\mathbf{1}^{\prime} & 0
\end{array}\right), \\
\mathbf{C} & \triangleq\left(\begin{array}{cc}
\mathbf{B} & \mathbf{B} \mathbf{1} \\
\mathbf{1}^{\prime} \mathbf{B} & 0
\end{array}\right), \\
\mathbf{B} & \triangleq \operatorname{diag}\left(\omega_{1} \mathbf{B}_{k+1}, \ldots, \omega_{N} \mathbf{B}_{k+N}\right),
\end{aligned}
$$

where $\mathbf{B}_{k+n}$ is defined by (47) for $n=1, \ldots, N$; $\mathbf{I}$ and $\mathbf{1}$ are an identity matrix and a "1" vector with compatible dimensions respectively. The problem (49) is an SDP problem. The derivation of the problem (49) is given in Appendix.

Note that the procedure for generating a feasible solution of the problem (45) from the solution of the problem (49) is also problem dependent, i.e., relying on the equalities or inequalities (32) and the Boolean constraint. As an illustration, let us again consider the representative constraints (21), (22) and (40). Thus, the specific expressions of $\mathbf{a}_{p}$ and $b_{p}$ in the optimization problem (49) are given by (42) and (43).

Based on the SDP (49), a typical Gaussian randomization procedure is used to construct an approximate solution to the problem (45]) here (see [25]). Thus, we have the following algorithm.

Algorithm 5.1 (Sensor selection scheme with the energy constraint for correlated sensors).

- Step 1: Given an optimal solution of the SDP (49) $X^{*} \in \mathbb{S}^{(N L+1)}$, and a number of randomizations $S$.

- Step 2: Generate $S$ feasible solutions by Gaussian randomization procedure based on $X^{*}$ : for $s=1: S$

1. Generate a vector $\xi_{s} \sim \mathcal{N}\left(0, X^{*}\right)$. Set $\eta_{s} \triangleq \xi_{s}(1: N L)$ which means the first $N L$ entries of $\xi_{s}$.

2. Without loss of generality, assume that $\omega_{1} \leq \omega_{2} \leq \ldots \leq \omega_{N}$. We generate the selection scheme from the $N$-th time step to the first time step. Set the index set of candidate sensors $\dot{\mathrm{n}} \triangleq\{1, \ldots, L\}$.

* Iteratively generate $\gamma_{k+N-n, s}^{i}$ for the $(N-n)$-th time step, $n=0, \ldots,(N-1)$ for $n=0:(N-1)$

$$
\gamma_{k+N-n, s}^{i} \triangleq\left\{\begin{array}{ll}
1, & \text { if } i \in \dot{\mathbb{1}}_{1}, \\
0, & \text { if } i \in \dot{\mathbb{1}}_{2},
\end{array}, \text { for } i=1, \ldots, L,\right.
$$


where $\dot{\mathrm{i}}_{1}$ is the index set of the first $m_{k+N-n}$ maximum entries of $\eta_{s}(J(L-n-1)+1$ : $J(L-n))$ in the index set of candidate sensors $\dot{\mathrm{i}}$ and $\dot{\mathrm{i}}_{2}=\dot{\mathrm{i}}-\dot{\mathrm{i}}_{1}$. Set $m_{k}^{i}:=m_{k}^{i}-1$, for $i \in \dot{\mathrm{i}}_{1}$. Update the index set of candidate sensors $\dot{\mathrm{i}} \triangleq\left\{i: m_{k}^{i}>0, i=1, \ldots, L\right\}$. end

3. Denote $\gamma_{k+N-n, s} \triangleq\left(\gamma_{k+N-n, s}^{1}, \ldots, \gamma_{k+N-n, s}^{L}\right)$ and $\gamma_{s} \triangleq\left(\gamma_{k+1, s}, \ldots, \gamma_{k+N, s}\right)$.

end

- Step 3: Determine $s^{*}=\operatorname{argmax}_{s=1, \ldots, S} f\left(\gamma_{s}\right)$ where $f(\cdot)$ may be the objective funcrions $f_{1}, f_{2}$ or $f_{3}$ defined in (9), (10) and (34) respectively.

- Step 4: Output $\hat{\gamma}=\gamma_{s^{*}}$ as the sensor selections of the problem (45).

Note that specific design of the randomization procedure technique is problem dependent. Here, to construct the feasible solution satisfying the constraints (21), (22) and (40), we employ Equation (50). The choice of $S$ will be discussed in Section 6 , Based on simulations, the randomized solution can often achieve a good performance with a small $S$, which is similar to that in [25]. The main computational complexity of the algorithm is in Step 1 where an SDP problem needs to be solved. The SDP problem can be solved efficiently by using interior-point methods (see, e.g., [20]).

\section{Numerical Examples}

In this section, we present a number of illustrative examples. Both uncorrelated and correlated sensor measurement noise cases are considered.

\subsection{Uncorrelated sensor measurement noises}

We first compare the performance of the approach given in Theorem 4.1 with the one in Joshi an Boyd [3] and the one in Mo et al. [4].

Example 6.1. Let us consider a dynamic system with $L=40$ sensors which are uniformly distributed over a square of size $100 \mathrm{~m}$. The parameter matrices and noise covariances for the dynamic system (1)-(4) are

$$
\begin{aligned}
\mathbf{F}_{k} & =\left(\begin{array}{ll}
1 & 0 \\
0 & 1
\end{array}\right), \mathbf{Q}_{k}=\left(\begin{array}{cc}
5 & 0 \\
0 & 10
\end{array}\right), \\
\mathbf{H}_{k}^{i} & =\left(\begin{array}{ll}
1 & 0 \\
0 & 1
\end{array}\right), \mathbf{R}_{k}^{i}=\left(\begin{array}{cc}
r_{1}^{i} & 0 \\
0 & r_{2}^{i}
\end{array}\right), i=1, \ldots, L,
\end{aligned}
$$

where $r_{1}^{i}$ and $r_{2}^{i}$ are randomly sampled from the uniform distribution in [5, 7] and [10 12] respectively. We consider a constraint, i.e., select $m_{k+n}=[1,5,10,15,20]$ sensors from 40 sensors at the next time step respectively. 
In Figure 1, the traces of the estimation error covariance are plotted for the sensor selection method given in Theorem 4.1, the one in Joshi an Boyd [3] and the one in Mo et al. [4] respectively. The CPU time is plotted in Figure 2 for the three algorithms respectively. Figure 1 shows that the three methods obtained very close and similar estimation performance for the numerical example, while Figure 2 shows that the CPU time of the method in Theorem 4.1 is much smaller than that of the one in Joshi an Boyd [3] and the one in Mo et al. [4]. The reason is that the method in Theorem 4.1 is an analytical solution. In addition, the computation time of the three methods is not an increasing function of the number of selected sensors. The reason is that when the number of selected sensors increases, the number of the decision variables does not increase and the structure of the optimization does not change; only some parameters of the equality constraints are changed.

Moreover, we consider a representative target tracking dynamic system with energy constraints. We assume that each target will be tracked in a Cartesian frame. The four state variables include position and velocity $(x, \dot{x}, y, \dot{y})$ respectively (see e.g., [24]). The parameter matrices and noise covariances for the dynamic system (1)-(4) are

$$
\begin{aligned}
\mathbf{F}_{k} & =\left(\begin{array}{cccc}
1 & T & 0 & 0 \\
0 & 1 & 0 & 0 \\
0 & 0 & 1 & T \\
0 & 0 & 0 & 1
\end{array}\right), \mathbf{Q}_{k}=\left(\begin{array}{cccc}
T^{3} / 3 & T^{2} / 2 & 0 & 0 \\
T^{2} / 2 & T & 0 & 0 \\
0 & 0 & T^{3} / 3 & T^{2} / 2 \\
0 & 0 & T^{2} / 2 & T
\end{array}\right), \\
\mathbf{H}_{k}^{i} & =\left(\begin{array}{llll}
1 & 0 & 0 & 0 \\
0 & 0 & 1 & 0
\end{array}\right), \quad \mathbf{R}_{k}^{i}, i=1, \ldots, L,
\end{aligned}
$$

where $T=1 \mathrm{~s}$ is the sampling interval; $\mathbf{F}_{k}, \mathbf{Q}_{k}, \mathbf{H}_{k}^{i}$ are the same in the following examples. The difference is the noise covariance of measurements, $\mathbf{R}_{k}^{i}$, in the following examples. Since the algorithm in Joshi an Boyd [3] that requires the measurement matrix is full-column rank when the measurement matrices of each sensor are the same and the one in Mo et al. [4] does not present how to threshold the approximate solution to generate a feasible solution satisfying the energy constraints, we evaluate the performance of Algorithm 4.2 by comparing with the exhaustive method for a monitoring system which has a small number of sensors and using the gap given in the Step 3 of Algorithm 4.2 for a monitoring system which has a large number of sensors in the following examples respectively.

Example 6.2. First, to compare with the exhaustive method, let us consider a relatively small monitoring system with $L=9$ sensors which are uniformly distributed over a square of size $100 \mathrm{~m}$. The parameter matrices of the dynamic system are given in (53)-(54) where

$$
\mathbf{R}_{k}^{i}=\left(\begin{array}{cc}
r_{1}^{i} & 0 \\
0 & r_{2}^{i}
\end{array}\right),
$$

$r_{1}^{i}$ and $r_{2}^{i}$ are randomly sampled from the uniform distribution in [5, 10]. We consider the optimization problem (37) with temporally inseparable constraint (40) and the constraints (21), (22) where $N=3$, 
$m_{k+n}=2, n=1, \ldots, N$ and $m_{k}^{i}=2$, i.e., select 2 sensors from 9 sensors at each time step and select each sensor less than twice in 3 time steps.

In Figure 3, the traces of the final estimation error covariance $f_{1}$ based on the three methods are plotted respectively, where $r_{1}^{i}$ and $r_{2}^{i}$ are randomly sampled 50 times. The three methods are 1) the exhaustive method that minimizes the final estimation error covariance $f_{1}, 2$ ) Algorithm 4.2 that maximizes the weighted information measure $f_{3}$ with weights $[1 / 3,1 / 3,1 / 3]$ and 3) Algorithm 4.2 that maximizes the weighted information measure $f_{3}$ with weights $[0,0,1]$ respectively. Similarly, the traces of the average estimation error covariance $f_{2}$ are plotted in Figure 4. In Figure 5, the sum of information measures of $N$ time steps $\sum_{n=1}^{N} \operatorname{tr}\left(\tilde{\mathbf{H}}_{k+n}^{\prime} \tilde{\mathbf{R}}_{k+n}^{+} \tilde{\mathbf{H}}_{k+n}\right)$ is plotted for the two sensor selection schemes respectively. They are obtained from 2) and 3) respectively.

From Figures 3-5, we have following observations:

- Figure 3 shows that the trace of the final estimation error covariances obtained from Algorithm 4.2 with two different weights that maximizes the weighted information measure $f_{3}$ are very close to that of the exhaustive method. Similarly, Figure 4 shows that the trace of average estimation error covariance obtained from Algorithm 4.2 with weights $[1 / 3,1 / 3,1 / 3]$ that maximizes the weighted information measure $f_{3}$ is very close to that of the exhaustive method. These indicate that maximization of the weighted information measure $f_{3}$ is a good alternative criterion for minimizing final or average estimation error covariance for sensor selection.

- Moreover, in Figure 3, when the objective is minimization of the final estimation error covariance $f_{1}$, both Algorithm 4.2 with weights $[0,0,1]$ and Algorithm 4.2 with weights $[1 / 3,1 / 3,1 / 3]$ are near optimal for sensor selection. However, Figure 5 shows that the sum of information measures of $N$ time steps for Algorithm 4.2 with the weights $[1 / 3,1 / 3,1 / 3]$ is larger than that of Algorithm 4.2 with the weights $[0,0,1]$. Thus, it is better to choose the weights $[0,0,1]$, since a larger sum of information measures of $N$ time steps implies that more good sensors are used.

- Finally, Figure 4 shows that when the objective is to minimize the average estimation error covariance $f_{2}$, it is better to choose the weights $[1 / 3,1 / 3,1 / 3]$ since Algorithm 4.2 with the weights $[1 / 3,1 / 3,1 / 3]$ is near optimal.

Example 6.3. Next, let us consider a large monitoring system with $L=20 \times 20=400$ sensors which are uniformly distributed in a square of size $100 \mathrm{~m}$. We consider the optimization problem (37) with temporally inseparable constraint (40) and the constraints (21), (22) where $N=5, m_{k+n}=10, n=1, \ldots, N$ and $m_{k}^{i}=2$, i.e., select 10 sensors at each time step from 400 sensors and select each sensor less than twice in 5 time steps. Moreover, we consider the performance of Algorithm 4.2 for different cases of $m_{k+n}$ from 10 to 100. Obviously, the exhaustive method is infeasible.

In Figure 6, the upper bound and lower bound of the objective function of the optimization problem (37) are plotted based on 50 Monte Carlo runs. The corresponding gaps, i.e, the upper bound minus the lower 
bound are plotted in Figure 7 Figures 6 and 7 show that the gaps are very small and Algorithm 4.2 can obtain the optimal solution in the sense of maximizing the weighted information measure $f_{3}$ in many cases, although the sensor network is large where the Boolean decision variables are more than 2000. Figure 8 presents the gaps as a function of $m_{k+n}$ from 10 to 100. It shows that the gaps are increasing as the number of selected sensors.

\subsection{Correlated sensor measurement noises}

In this subsection, we will compare Algorithm 5.1 with the exhaustive method for a simple problem so that the approximation loss can be computed. For this, we assume that only the sensor selection scheme for the next step is to be designed, i.e., $N=1$. For $N=5$, we will compare Algorithm 5.1 with Algorithm 4.2 that ignores dependence. In this case, the exhaustive method is infeasible, since we have to enumerate $2.43 \times 10^{10}$ cases. At the end, an example that compares the root mean square error (RMSE) of state estimation based on sensor selection is presented.

Example 6.4. Let us consider $L=25$ for the sensor network shown in Figure 14 Assume that there is a jammer signal $\mathbf{v}_{k}^{0}$ with a covariance $\mathbf{R}_{k}^{0}$ at the position $(550 \mathrm{~m}, 200 \mathrm{~m})$, besides the natural noises $\mathbf{v}_{k}^{i}, i=$ $1, \ldots, L$ which are independent of $\mathbf{v}_{k}^{0}$. The jamming signal introduces dependence among measurement noises. Thus, the noises at the $i$-th sensor is given as follows

$$
\breve{\mathbf{v}}_{k}^{i}=\mathbf{v}_{k}^{i}+\frac{P_{0}}{1+\alpha d_{i, 0}^{n}} \mathbf{v}_{k}^{0}=\mathbf{v}_{k}^{i}+\beta_{i} \mathbf{v}_{k}^{0}
$$

where $\beta_{i} \triangleq \frac{P_{0}}{1+\alpha d_{i, 0}^{n}} ; d_{i, 0}$ is the distance between the jammer and the $i$-th sensor; the signal decay exponent $n=2$, the scaling parameter $\alpha=1$ and different values for the signal power $P_{0}=[1,3,6,10,12,15,20] \times$ $10^{5}$ are used in simulations respectively. Thus, noises of sensors are correlated and the $i$-th block and $j$-th block of the noise covariance $\breve{\mathbf{R}}_{k}$ can be computed by (56) to be

$$
\breve{\mathbf{R}}_{k}^{i j}=\operatorname{Cov}\left(\breve{\mathbf{v}}_{k}^{i}, \breve{\mathbf{v}}_{k}^{j}\right)=\mathbf{R}_{k}^{i j}+\beta_{i} \beta_{j} \mathbf{R}_{k}^{0}
$$

where

$$
\mathbf{R}_{k}^{i j}=\left\{\begin{array}{l}
\left(\begin{array}{cc}
10 & 0 \\
0 & 10
\end{array}\right), \quad \text { if } i=j \\
\left(\begin{array}{cc}
0 & 0 \\
0 & 0
\end{array}\right), \\
i \neq j .
\end{array} \quad, \quad \mathbf{R}_{k}^{0}=\left(\begin{array}{cc}
1 & 0 \\
0 & 1
\end{array}\right)\right.
$$

are used in simulations. Note that the corresponding Pearson's correlation coefficients between sensors are approximately equal to $[0.1,0.2,0.4,0.6,0.7,0.8,0.9]$ corresponding to $P_{0}=[1,3,6,10,12,15,20] \times$ $10^{5}$ respectively. We consider the optimization problem (45) with temporally separable constraints (21), (22) where $N=1, m_{k+1}=2$, i.e., select 2 sensors from 25 sensors at the next time step. 
In Figure 9, comparisons of the objective function $\operatorname{tr}\left(\tilde{\mathbf{H}}_{k+1}^{\prime} \tilde{\mathbf{R}}_{k+1}^{+} \tilde{\mathbf{H}}_{k+1}\right)$ of the optimization problem (45) (i.e., the information measure of the $(k+1)$-th time step) based on the exhaustive method, Algorithm 5.1 and Theorem 4.1 that ignores dependence are plotted for different jammer signal powers respectively. We present the performance of Algorithm 5.1 with small numbers of randomizations $S=20, S=100$. Similarly, comparisons of the traces of the estimation error covariance of $(k+1)$-th time step are plotted in Figure 10 .

From Figures 9-10, we have the following observations:

- For all the three methods, the larger is the signal power of jammer, the smaller is the information measure of the $(k+1)$-th time step obtained from the selected sensors and the larger is the trace of the estimation error covariance at the $(k+1)$-th time step.

- Figures $9-10$ also show that the exhaustive method yields better results than Algorithm 5.1 with small $S$ and the latter is better than the method of Theorem 4.1 that ignores dependence, especially in the case of strong dependence (i.e., strong signal power of the jammer).

- Figures $9-10$ indicate that larger the value of $S$ is, the closer is the performance of Algorithm 5.1 to that of the exhaustive method, i.e., the smaller is the approximation loss of Algorithm 5.1 .

Example 6.5. Next, let us consider a monitoring system with a large $N=5$ and $L=5 \times 5=25$ sensors which are uniformly distributed in a square of size $100 \mathrm{~m}$. We consider the optimization problem (37) with temporally inseparable constraint (40) and the constraints (21), (22) where $m_{k+n}=2, n=1, \ldots, N$ and $m_{k}^{i}=2$, i.e., select 2 sensors at each time step from 25 sensors and select each sensor less than twice in next 5 time steps.

In Figure 11, comparisons of the objective function $\sum_{n=1}^{N} \omega_{n} \operatorname{tr}\left(\tilde{\mathbf{H}}_{k+n}^{\prime} \tilde{\mathbf{R}}_{k+n}^{+} \tilde{\mathbf{H}}_{k+n}\right)$ of the optimization problem (45) (i.e., the sum of the weighted information measure of $N$ time steps, $f_{3}$ defined in (34)) based on approaches of Algorithm 5.1 and Algorithm 4.2 that ignores dependence are plotted for different jammer signal powers respectively. We examine the performance of Algorithm 5.1 as a function of the number of randomizations $S=20, S=100, S=2000$ which are small, compared with the exhaustive number $2.43 \times 10^{10}$. Similarly, comparisons of the traces of the average estimation error covariances of $N$ time steps are plotted in Figure 12

Figures 11 12 show that Algorithm 5.1 with a small value of $S$ is better than Algorithm 4.2 that ignores dependence, especially in the case of strong dependence (i.e., strong signal power of the jammer). In addition, Figures 11-12 indicate that larger the value of $S$ is, the better is the performance of Algorithm 5.1 than that of Algorithm 4.2 that ignores dependence.

Example 6.6. Finally, let us consider the L-sensor noise covariance $\mathbf{R}_{k}, k=1,2, \ldots$ which depends on the state $\mathbf{x}_{k}$. A frequently made assumption is that larger is the distance between the sensor and the target, larger is the noise covariance. However, when we design the sensor selection scheme of next $N$ time steps at 
time $k$, we do not know the state $\mathbf{x}_{k+n}$ so that we replace it by the state prediction $\mathbf{x}_{k+n \mid k}$ which is used to compute the $\mathbf{R}_{k+n}, n=1, \ldots, N$. Specifically, the noise covariance $\mathbf{R}_{k+n}$ is

$$
\mathbf{R}_{k+n}=\overline{\mathbf{R}}_{k+n}+\breve{\mathbf{R}}_{k+n}
$$

where $\breve{\mathbf{R}}_{k+n}$ is the noise covariance from the jammer signal $\mathbf{v}_{k}^{0}$ defined in (57) and the signal power of jammer $P_{0}=1.5 \times 10^{6} ; \overline{\mathbf{R}}_{k+n}$ is a diagonal matrix with the $i$-th diagonal block defined as follows

$$
\overline{\mathbf{R}}_{k+n}^{i i}=\left(\begin{array}{cc}
\alpha_{1} d_{i, n} & 0 \\
0 & \alpha_{1} d_{i, n}
\end{array}\right), i=1, \ldots, L, n=1, \ldots, N,
$$

where $\alpha_{1}=0.05$ is a scaling parameter; $d_{i, n}$ is the distance between the target prediction $\mathbf{x}_{k+n \mid k}$ and the $i$-th sensor. We consider the optimization problem (37) with temporally separable constraint (40) and the constraints (21), (22) where $N=5, m_{k+n}=2, n=1, \ldots, N$ and $m_{k}^{i}=2$, i.e., select 2 sensors at each time step and select each sensor less than twice in 5 time steps. The initial state of the target is $(600 \mathrm{~m},-20 \mathrm{~m} / \mathrm{s}, 200 \mathrm{~m}, 0 \mathrm{~m} / \mathrm{s})$.

In Figure 13, RMSE of the state estimates based on 200 Monte Carlo runs is given. We compare Algorithm 4.2 that ignores dependence with Algorithm 5.1 with $S=20, S=100$ and $S=2000$ respectively.

As far as the RMSE is considered, Figure 13 also shows that Algorithm 5.1 with a small value of $S$ is better than Algorithm 4.2 that ignores dependence and that larger the value of $S$ is, the better is the performance of Algorithm 5.1 than that of Algorithm 4.2 that ignores dependence.

\section{Conclusion}

In this paper, we have proposed a generalized information filter for target tracking in wireless sensor networks where measurements from a subset of sensors are employed at each time step. Then, under a regularity condition, we proved that the multistage look-ahead policy that minimizes either the final or the average estimation error covariances of next $N$ time steps is equivalent to the myopic sensor selection policy that maximizes the trace of the generalized information gain at each time step. When the measurement noises are uncorrelated, the optimal solution has been derived analytically for sensor selection with temporally separable constraints. For temporally inseparable constraints, the sensor selection scheme can be obtained by approximately solving an LP problem. Although there is no guarantee that the gap between the performance of the chosen subset and the performance bound is always small, numerical examples showed that the algorithm is nearoptimal in many cases and the selection scheme for a large sensor network with more than 2000 Boolean decision variables can be dealt with quickly. Finally, when the noises of measurements are correlated, the sensor selection problem with temporally inseparable constraints was relaxed to a BQP problem which can be efficiently solved by a Gaussian randomization procedure by solving an SDP problem which can be solved by interior-point methods and related software tools. Numerical examples showed that the proposed method is much better than the method that ignores dependence. 
Future work will involve the generalization from the linear dynamic systems to nonlinear dynamic systems. The equivalence between multistage look-ahead optimization policy for sensor management and the myopic sensor optimization policy and the corresponding sensor management schemes will be investigated. In addition, it can be considered for various applications such as robotics, sensor placement for structures and different types of wireless networks.

\section{Appendix}

\section{The proof of Theorem 2.1 ,}

Proof. Notice that there are $m_{k+1}$ number of $\gamma_{k+1}^{i}=1$ and $L-m_{k+1}$ number of $\gamma_{k+1}^{i}=0$ so that there exists a permutation matrix $\mathbf{P}$ such that

$$
\mathbf{P} \tilde{\mathbf{H}}_{k+1}=\left(\begin{array}{c}
\mathbf{H}_{k+1}\left(1: m_{k+1}\right) \\
\mathbf{0}
\end{array}\right)
$$

and

$$
\mathbf{P} \tilde{\mathbf{R}}_{k+1} \mathbf{P}^{\prime}=\left(\begin{array}{cc}
\mathbf{R}_{k+1}\left(1: m_{k+1}\right) & \mathbf{0} \\
\mathbf{0} & \mathbf{0}
\end{array}\right)
$$

where $\mathbf{H}_{k+1}\left(1: m_{k+1}\right)$ and $\mathbf{R}_{k+1}\left(1: m_{k+1}\right)$ are the stacked measurement matrices and the covariance of noises of the $m_{k+1}$ selected sensor respectively; $\mathbf{0}$ is a zero matrix with compatible dimensions. From the property of the permutation matrix $\mathbf{P} \times \mathbf{P}^{\prime}=\mathbf{P}^{\prime} \times \mathbf{P}=\mathbf{I}$ and the definition of Moore-Penrose inverse, we have

$$
\begin{aligned}
\mathbf{P} \tilde{\mathbf{R}}_{k+1}^{+} \mathbf{P}^{\prime} & =\left(\mathbf{P} \tilde{\mathbf{R}}_{k+1} \mathbf{P}^{\prime}\right)^{+} \\
& =\left(\begin{array}{cc}
\mathbf{R}_{k+1}\left(1: m_{k+1}\right) & \mathbf{0} \\
\mathbf{0} & \mathbf{0}
\end{array}\right)^{+} \\
& =\left(\begin{array}{cc}
\left(\mathbf{R}_{k+1}\left(1: m_{k+1}\right)\right)^{-1} & \mathbf{0} \\
\mathbf{0} & \mathbf{0}
\end{array}\right)
\end{aligned}
$$


Moreover, by equations (60)-(62) and repeatedly using the definition of Moore-Penrose generalized inverse and the property of the permutation matrix $\mathbf{P} \times \mathbf{P}^{\prime}=\mathbf{P}^{\prime} \times \mathbf{P}=\mathbf{I}$, we have the following derivation

$$
\begin{aligned}
& \left(\tilde{\mathbf{R}}_{k+1}+\tilde{\mathbf{H}}_{k+1} \mathbf{P}_{k+1 \mid k} \tilde{\mathbf{H}}_{k+1}^{\prime}\right)^{+} \\
& =\left(\mathbf{P}^{\prime}\left(\begin{array}{cc}
\mathbf{R}_{k+1}\left(1: m_{k+1}\right) & \mathbf{0} \\
\mathbf{0} & \mathbf{0}
\end{array}\right) \mathbf{P}\right. \\
& \left.+\mathbf{P}^{\prime}\left(\begin{array}{cc}
\left.\tilde{\mathbf{H}}_{k+1}\left(1: m_{k+1}\right) \mathbf{P}_{k+1 \mid k} \tilde{\mathbf{H}}_{k+1}\left(1: m_{k+1}\right)^{\prime}\right) & \mathbf{0} \\
\mathbf{0} & \mathbf{0}
\end{array}\right) \mathbf{P}\right)^{+} \\
& =\left(\mathbf{P}^{\prime}\left(\begin{array}{cc}
\left.\mathbf{R}_{k+1}\left(1: m_{k+1}\right)+\tilde{\mathbf{H}}_{k+1}\left(1: m_{k+1}\right) \mathbf{P}_{k+1 \mid k} \tilde{\mathbf{H}}_{k+1}\left(1: m_{k+1}\right)^{\prime}\right) & \mathbf{0} \\
\mathbf{0} & \mathbf{0}
\end{array}\right) \mathbf{P}\right)^{+} \\
& =\mathbf{P}^{\prime}\left(\begin{array}{cc}
\left(\mathbf{R}_{k+1}\left(1: m_{k+1}\right)+\tilde{\mathbf{H}}_{k+1}\left(1: m_{k+1}\right) \mathbf{P}_{k+1 \mid k} \tilde{\mathbf{H}}_{k+1}\left(1: m_{k+1}\right)^{\prime}\right) & \mathbf{0} \\
\mathbf{0} & \mathbf{0}
\end{array}\right)^{+} \mathbf{P} \\
& =\mathbf{P}^{\prime}\left(\begin{array}{cc}
\left(\mathbf{R}_{k+1}\left(1: m_{k+1}\right)+\tilde{\mathbf{H}}_{k+1}\left(1: m_{k+1}\right) \mathbf{P}_{k+1 \mid k} \tilde{\mathbf{H}}_{k+1}\left(1: m_{k+1}\right)^{\prime}\right)^{-1} & \mathbf{0} \\
\mathbf{0} & \mathbf{0}
\end{array}\right) \mathbf{P} \\
& =\mathbf{P}^{\prime}\left(\begin{array}{cc}
\left(\mathbf{R}_{k+1}\left(1: m_{k+1}\right)\right)^{-1}\left(\mathbf{I}+\tilde{\mathbf{H}}_{k+1}\left(1: m_{k+1}\right)\right. & \mathbf{0} \\
\left.\cdot \mathbf{P}_{k+1 \mid k} \tilde{\mathbf{H}}_{k+1}\left(1: m_{k+1}\right)^{\prime}\left(\mathbf{R}_{k+1}\left(1: m_{k+1}\right)\right)^{-1}\right)^{-1} & \\
\mathbf{0} & \mathbf{0}
\end{array}\right) \mathbf{P} \\
& =\mathbf{P}^{\prime}\left(\begin{array}{cc}
\left(\mathbf{R}_{k+1}\left(1: m_{k+1}\right)\right)^{-1} & \mathbf{0} \\
\mathbf{0} & \mathbf{0}
\end{array}\right) \\
& \cdot\left(\begin{array}{cc}
\left(\mathbf{I}+\tilde{\mathbf{H}}_{k+1}\left(1: m_{k+1}\right) \mathbf{P}_{k+1 \mid k} \tilde{\mathbf{H}}_{k+1}\left(1: m_{k+1}\right)^{\prime}\left(\mathbf{R}_{k+1}\left(1: m_{k+1}\right)\right)^{-1}\right)^{-1} & \mathbf{0} \\
\mathbf{0} & \mathbf{I}
\end{array}\right) \mathbf{P}
\end{aligned}
$$




$$
\begin{aligned}
& =\mathbf{P}^{\prime}\left(\begin{array}{cc}
\left(\mathbf{R}_{k+1}\left(1: m_{k+1}\right)\right)^{-1} & \mathbf{0} \\
\mathbf{0} & \mathbf{0}
\end{array}\right) \\
& \cdot\left(\mathbf{I}+\left(\begin{array}{cc}
\tilde{\mathbf{H}}_{k+1}\left(1: m_{k+1}\right) \mathbf{P}_{k+1 \mid k} \tilde{\mathbf{H}}_{k+1}\left(1: m_{k+1}\right)^{\prime} & \mathbf{0} \\
\mathbf{0} & \mathbf{0}
\end{array}\right)\right. \\
& \left.\left(\begin{array}{cc}
\left(\mathbf{R}_{k+1}\left(1: m_{k+1}\right)\right)^{-1} & \mathbf{0} \\
\mathbf{0} & \mathbf{0}
\end{array}\right)\right)^{-1} \mathbf{P} \\
& =\mathbf{P}^{\prime}\left(\begin{array}{cc}
\left(\mathbf{R}_{k+1}\left(1: m_{k+1}\right)\right)^{-1} & \mathbf{0} \\
\mathbf{0} & \mathbf{0}
\end{array}\right) \\
& \cdot\left(\mathbf{P}^{\prime}+\mathbf{P}^{\prime}\left(\begin{array}{cc}
\tilde{\mathbf{H}}_{k+1}\left(1: m_{k+1}\right) \mathbf{P}_{k+1 \mid k} \tilde{\mathbf{H}}_{k+1}\left(1: m_{k+1}\right)^{\prime} & \mathbf{0} \\
\mathbf{0} & \mathbf{0}
\end{array}\right)\right. \\
& \left.\cdot\left(\begin{array}{cc}
\left(\mathbf{R}_{k+1}\left(1: m_{k+1}\right)\right)^{-1} & \mathbf{0} \\
\mathbf{0} & \mathbf{0}
\end{array}\right)\right)^{-1} \\
& =\mathbf{P}^{\prime}\left(\begin{array}{cc}
\left(\mathbf{R}_{k+1}\left(1: m_{k+1}\right)\right)^{-1} & \mathbf{0} \\
\mathbf{0} & \mathbf{0}
\end{array}\right) \\
& \left(\mathbf{P}^{\prime}+\mathbf{P}^{\prime}\left(\begin{array}{cc}
\tilde{\mathbf{H}}_{k+1}\left(1: m_{k+1}\right) \mathbf{P}_{k+1 \mid k} \tilde{\mathbf{H}}_{k+1}\left(1: m_{k+1}\right)^{\prime} & \mathbf{0} \\
\mathbf{0} & \mathbf{0}
\end{array}\right) \mathbf{P} \tilde{\mathbf{R}}_{k+1}^{+} \mathbf{P}^{\prime}\right)^{-1} \\
& =\mathbf{P}^{\prime}\left(\begin{array}{cc}
\left(\mathbf{R}_{k+1}\left(1: m_{k+1}\right)\right)^{-1} & \mathbf{0} \\
\mathbf{0} & \mathbf{0}
\end{array}\right)\left(\mathbf{P}^{\prime}+\mathbf{P}^{\prime} \mathbf{P} \tilde{\mathbf{H}}_{k+1} \mathbf{P}_{k+1 \mid k} \tilde{\mathbf{H}}_{k+1}^{\prime} \mathbf{P}^{\prime} \mathbf{P} \tilde{\mathbf{R}}_{k+1}^{+} \mathbf{P}^{\prime}\right)^{-1} \\
& =\mathbf{P}^{\prime}\left(\begin{array}{cc}
\left(\mathbf{R}_{k+1}\left(1: m_{k+1}\right)\right)^{-1} & \mathbf{0} \\
\mathbf{0} & \mathbf{0}
\end{array}\right)\left(\mathbf{P}^{\prime}+\tilde{\mathbf{H}}_{k+1} \mathbf{P}_{k+1 \mid k} \tilde{\mathbf{H}}_{k+1}^{\prime} \tilde{\mathbf{R}}_{k+1}^{+} \mathbf{P}^{\prime}\right)^{-1} \\
& =\mathbf{P}^{\prime}\left(\begin{array}{cc}
\left(\mathbf{R}_{k+1}\left(1: m_{k+1}\right)\right)^{-1} & \mathbf{0} \\
\mathbf{0} & \mathbf{0}
\end{array}\right) \mathbf{P}\left(\mathbf{I}+\tilde{\mathbf{H}}_{k+1} \mathbf{P}_{k+1 \mid k} \tilde{\mathbf{H}}_{k+1}^{\prime} \tilde{\mathbf{R}}_{k+1}^{+}\right)^{-1} \\
& =\tilde{\mathbf{R}}_{k+1}^{+}\left(\mathbf{I}+\tilde{\mathbf{H}}_{k+1} \mathbf{P}_{k+1 \mid k} \tilde{\mathbf{H}}_{k+1}^{\prime} \tilde{\mathbf{R}}_{k+1}^{+}\right)^{-1} .
\end{aligned}
$$

From Equations (14) and (63), we have

$$
\begin{aligned}
\mathbf{K}_{k+1} & =\mathbf{P}_{k+1 \mid k} \mathbf{H}_{k+1}^{\prime}\left(\tilde{\mathbf{R}}_{k+1}+\tilde{\mathbf{H}}_{k+1} \mathbf{P}_{k+1 \mid k} \tilde{\mathbf{H}}_{k+1}^{\prime}\right)^{+} \\
& =\mathbf{P}_{k+1 \mid k} \mathbf{H}_{k+1}^{\prime} \tilde{\mathbf{R}}_{k+1}^{+}\left(\mathbf{I}+\tilde{\mathbf{H}}_{k+1} \mathbf{P}_{k+1 \mid k} \tilde{\mathbf{H}}_{k+1}^{\prime} \tilde{\mathbf{R}}_{k+1}^{+}\right)^{-1},
\end{aligned}
$$


which yields

$$
\mathbf{K}_{k+1}\left(\mathbf{I}+\tilde{\mathbf{H}}_{k+1} \mathbf{P}_{k+1 \mid k} \tilde{\mathbf{H}}_{k+1}^{\prime} \tilde{\mathbf{R}}_{k+1}^{+}\right)=\mathbf{P}_{k+1 \mid k} \mathbf{H}_{k+1}^{\prime} \tilde{\mathbf{R}}_{k+1}^{+},
$$

so that

$$
\mathbf{K}_{k+1} \mathbf{I}+\mathbf{K}_{k+1} \tilde{\mathbf{H}}_{k+1} \mathbf{P}_{k+1 \mid k} \tilde{\mathbf{H}}_{k+1}^{\prime} \tilde{\mathbf{R}}_{k+1}^{+}=\mathbf{P}_{k+1 \mid k} \mathbf{H}_{k+1}^{\prime} \tilde{\mathbf{R}}_{k+1}^{+} .
$$

Moreover, we have

$$
\begin{aligned}
\mathbf{K}_{k+1} & =\left(\mathbf{I}-\mathbf{K}_{k+1} \tilde{\mathbf{H}}_{k+1}\right) \mathbf{P}_{k+1 \mid k} \mathbf{H}_{k+1}^{\prime} \tilde{\mathbf{R}}_{k+1}^{+} \\
& =\mathbf{P}_{k+1 \mid k+1} \mathbf{H}_{k+1}^{\prime} \tilde{\mathbf{R}}_{k+1}^{+},
\end{aligned}
$$

where the derivation of (64) is based on (12).

From Equations (12) and (64), we have

$$
\begin{aligned}
\mathbf{P}_{k+1 \mid k}^{-1} & =\mathbf{P}_{k+1 \mid k+1}^{-1}\left(\mathbf{I}-\mathbf{K}_{k+1} \tilde{\mathbf{H}}_{k+1}\right), \\
& =\mathbf{P}_{k+1 \mid k+1}^{-1}-\mathbf{P}_{k+1 \mid k+1}^{-1} \mathbf{K}_{k+1} \tilde{\mathbf{H}}_{k+1}, \\
& =\mathbf{P}_{k+1 \mid k+1}^{-1}-\mathbf{H}_{k+1}^{\prime} \tilde{\mathbf{R}}_{k+1}^{+} \tilde{\mathbf{H}}_{k+1} .
\end{aligned}
$$

Thus, we have $\mathbf{P}_{k+1 \mid k+1}^{-1}=\mathbf{P}_{k+1 \mid k}^{-1}+\mathbf{H}_{k+1}^{\prime} \tilde{\mathbf{R}}_{k+1}^{+} \tilde{\mathbf{H}}_{k+1}$.

By (11), (12) and (64),

$$
\begin{aligned}
\mathbf{x}_{k+1 \mid k+1} & =\left(\mathbf{I}-\mathbf{K}_{k+1} \tilde{\mathbf{H}}_{k+1}\right) \mathbf{x}_{k+1 \mid k}+\mathbf{K}_{k+1} \mathbf{z}_{k+1}, \\
& =\left(\mathbf{I}-\mathbf{K}_{k+1} \tilde{\mathbf{H}}_{k+1}\right) \mathbf{x}_{k+1 \mid k}+\mathbf{P}_{k+1 \mid k+1} \mathbf{H}_{k+1}^{\prime} \tilde{\mathbf{R}}_{k+1}^{+} \mathbf{z}_{k+1}, \\
& =\mathbf{P}_{k+1 \mid k+1} \mathbf{P}_{k+1 \mid k}^{-1} \mathbf{x}_{k+1 \mid k}+\mathbf{P}_{k+1 \mid k+1} \mathbf{H}_{k+1}^{\prime} \tilde{\mathbf{R}}_{k+1}^{+} \mathbf{z}_{k+1}, \\
& =\mathbf{P}_{k+1 \mid k+1}\left\{\mathbf{P}_{k+1 \mid k}^{-1} \mathbf{x}_{k+1 \mid k}+\mathbf{H}_{k+1}^{\prime} \tilde{\mathbf{R}}_{k+1}^{+} \mathbf{z}_{k+1}\right\},
\end{aligned}
$$

Thus, we have $\mathbf{x}_{k+1 \mid k+1}=\mathbf{P}_{k+1 \mid k+1}\left\{\mathbf{P}_{k+1 \mid k}^{-1} \mathbf{x}_{k+1 \mid k}+\tilde{\mathbf{H}}_{k+1}^{\prime} \tilde{\mathbf{R}}_{k+1}^{+} \mathbf{z}_{k+1}\right\}$.

\section{The proof of Lemma 3.1 .}

Proof. First, we prove that the optimal solution of $\left(A_{1}\right)$ is also the optimal solution of $\left(A_{2}\right)$. If $\mathbf{x}_{1}$ is the optimal solution of $\left(A_{1}\right)$, then, for arbitrary $\mathbf{x} \in \mathcal{S}, \mathbf{M}(\mathbf{x}) \preceq \mathbf{M}\left(\mathbf{x}_{1}\right)$ which yields $\operatorname{tr}(\mathbf{M}(\mathbf{x})) \leq \operatorname{tr}\left(\mathbf{M}\left(\mathbf{x}_{1}\right)\right)$. Thus, $\mathbf{x}_{1}$ is also the optimal solution of $\left(A_{2}\right)$.

On the other hand, if $\mathbf{x}_{2}$ is the optimal solution of $\left(A_{2}\right)$, then, for arbitrary $\mathbf{x} \in \mathcal{S}, \operatorname{tr}(\mathbf{M}(\mathbf{x})) \leq$ $\operatorname{tr}\left(\mathbf{M}\left(\mathbf{x}_{2}\right)\right)$ which implies $\operatorname{tr}\left(\mathbf{M}\left(\mathbf{x}_{1}\right)\right) \leq \operatorname{tr}\left(\mathbf{M}\left(\mathbf{x}_{2}\right)\right)$. Notice that the problem $\left(A_{1}\right)$ has an optimal solution $\mathbf{x}_{1}$ which yields $\operatorname{tr}\left(\mathbf{M}\left(\mathbf{x}_{2}\right)\right) \leq \operatorname{tr}\left(\mathbf{M}\left(\mathbf{x}_{1}\right)\right)$. Thus, $\operatorname{tr}\left(\mathbf{M}\left(\mathbf{x}_{1}\right)\right)=\operatorname{tr}\left(\mathbf{M}\left(\mathbf{x}_{2}\right)\right)$ so that $\operatorname{tr}\left(\mathbf{M}\left(\mathbf{x}_{1}\right)-\mathbf{M}\left(\mathbf{x}_{2}\right)\right)=0$. By $\operatorname{tr}\left(\mathbf{M}\left(\mathbf{x}_{1}\right)-\mathbf{M}\left(\mathbf{x}_{2}\right)\right)=0$ and $\mathbf{M}\left(\mathbf{x}_{1}\right)-\mathbf{M}\left(\mathbf{x}_{2}\right) \succeq 0$, we have $\mathbf{M}\left(\mathbf{x}_{1}\right)=\mathbf{M}\left(\mathbf{x}_{2}\right)$. Therefore, $\mathbf{x}_{2}$ is also the optimal solution of $\left(A_{1}\right)$. 


\section{The proof of Lemma 3.2 ,}

Proof. In one direction: if $\mathbf{x}_{1}^{*}, \ldots, \mathbf{x}_{N}^{*}$ is the optimal solution of $\left(\left(B_{1}\right)\right.$ with $\left.n=N\right)$, then, for $n=1, \ldots, N$,

$$
\mathbf{M}_{n}\left(\mathbf{x}_{1}^{*}, \ldots, \mathbf{x}_{n}^{*}\right) \preceq \mathbf{M}_{n}\left(\mathbf{x}_{1}, \ldots, \mathbf{x}_{n}\right) \text { for arbitrary } \mathbf{x}_{i} \in \mathcal{S}_{i}, i=1, \ldots, n
$$

since the optimal solution that minimizes $\mathbf{M}_{n}\left(\mathbf{x}_{1}, \ldots, \mathbf{x}_{n}\right)$ is the same as that minimizes $\mathbf{M}_{n+1}\left(\mathbf{x}_{1}, \ldots, \mathbf{x}_{n+1}\right)$, for $n=1, \ldots, N$. Thus,

$$
\sum_{n=1}^{N} \mathbf{M}_{n}\left(\mathbf{x}_{1}^{*}, \ldots, \mathbf{x}_{n}^{*}\right) \preceq \sum_{n=1}^{N} \mathbf{M}_{n}\left(\mathbf{x}_{1}, \ldots, \mathbf{x}_{n}\right) \text { for arbitrary } \mathbf{x}_{i} \in \mathcal{S}_{i}, i=1, \ldots, n,
$$

which yields $\mathbf{x}_{1}^{*}, \ldots, \mathbf{x}_{N}^{*}$ is also the optimal solution of $\left(B_{2}\right)$.

On the other hand, assume that $\mathbf{x}_{1}^{*}, \ldots, \mathbf{x}_{N}^{*}$ is the optimal solution of $\left(B_{2}\right)$. If $\mathbf{x}_{1}^{*}, \ldots, \mathbf{x}_{N}^{*}$ is not the optimal solution of $\left(B_{1}\right)$, then there exists an optimal solution $\mathbf{x}_{1}^{0}, \ldots, \mathbf{x}_{N}^{0}$ which has a smaller objective function value than that of $\mathbf{x}_{1}^{*}, \ldots, \mathbf{x}_{N}^{*}$. Since the optimal solution that minimizes $\mathbf{M}_{n}\left(\mathbf{x}_{1}, \ldots, \mathbf{x}_{n}\right)$ is the same as that minimizes $\mathbf{M}_{n+1}\left(\mathbf{x}_{1}, \ldots, \mathbf{x}_{n+1}\right), \mathbf{x}_{1}^{0}, \ldots, \mathbf{x}_{n}^{0}$ is the optimal solution of $\mathbf{M}_{n}\left(\mathbf{x}_{1}, \ldots, \mathbf{x}_{n}\right)$ for $n=1, \ldots, N$. Thus,

$$
\mathbf{M}_{n}\left(\mathbf{x}_{1}^{*}, \ldots, \mathbf{x}_{n}^{*}\right) \succeq \mathbf{M}_{n}\left(\mathbf{x}_{1}^{0}, \ldots, \mathbf{x}_{n}^{0}\right), n=1, \ldots, N
$$

so that

$$
\sum_{n=1}^{N} \mathbf{M}_{n}\left(\mathbf{x}_{1}^{*}, \ldots, \mathbf{x}_{n}^{*}\right) \succeq \sum_{n=1}^{N} \mathbf{M}_{n}\left(\mathbf{x}_{1}^{0}, \ldots, \mathbf{x}_{n}^{0}\right),
$$

which yields a contradiction. Thus, $\mathbf{x}_{1}^{*}, \ldots, \mathbf{x}_{N}^{*}$ is the optimal solution of $\left(B_{1}\right)$.

\section{The proof of Lemma 3.3 .}

Proof. If the problem (20) has an optimal solution, from the fact that any positive definite matrix $A \succeq A_{0}$ implies $A^{-1} \preceq A_{0}^{-1}$, then we have that the problem (20) is equivalent to solve

$$
\begin{aligned}
& \max _{\gamma_{k+n}^{i}} \quad\left(\mathbf{P}_{k+N \mid k+N}\left(\gamma_{k+1}^{1}, \ldots, \gamma_{k+1}^{L}, \ldots, \gamma_{k+N}^{1}, \ldots, \gamma_{k+N}^{L}\right)\right)^{-1} \\
&=\mathbf{P}_{k+N \mid k+N-1}^{-1}+\tilde{\mathbf{H}}_{k+N}^{\prime} \tilde{\mathbf{R}}_{k+N}^{+} \tilde{\mathbf{H}}_{k+N} \\
& \text { subject to } \quad \sum_{i=1}^{L} \gamma_{k+n}^{i}=m_{k+n}, n=1, \ldots, N, \\
& \gamma_{k+n}^{i} \in\{0,1\}, i=1,2, \ldots, L, n=1, \ldots, N,
\end{aligned}
$$


which has the same optimal solution. Since the constraints are temporally separable, and by Equation (15) and the invertibility of $\mathbf{F}_{k}$, it is equivalent to solve the following two problems

$$
\begin{aligned}
\max _{\gamma_{k+n}^{i}} & \tilde{\mathbf{H}}_{k+N}^{\prime} \tilde{\mathbf{R}}_{k+N}^{+} \tilde{\mathbf{H}}_{k+N} \\
\text { subject to } & \sum_{i=1}^{L} \gamma_{k+n}^{i}=m_{k+n}, n=N, \\
& \gamma_{k+n}^{i} \in\{0,1\}, i=1,2, \ldots, L, n=N,
\end{aligned}
$$

and

$$
\begin{aligned}
\min _{\gamma_{k+n}^{i}} & \mathbf{P}_{k+N-1 \mid k+N-1}\left(\gamma_{k+1}^{1}, \ldots, \gamma_{k+1}^{L}, \ldots, \gamma_{k+N-1}^{1}, \ldots, \gamma_{k+N-1}^{L}\right) \\
& =\left(\mathbf{P}_{k+N-1 \mid k+N-2}^{-1}+\tilde{\mathbf{H}}_{k+N-1}^{\prime} \tilde{\mathbf{R}}_{k+N-1}^{+} \tilde{\mathbf{H}}_{k+N-1}\right)^{-1} \\
\text { subject to } \quad & \sum_{i=1}^{L} \gamma_{k+n}^{i}=m_{k+n}, n=1, \ldots, N,-1, \\
& \gamma_{k+n}^{i} \in\{0,1\}, i=1,2, \ldots, L, n=1, \ldots, N,-1 .
\end{aligned}
$$

Both of them have an optimal solution respectively.

After $N$-step recursive decomposition, the problem (20) is equivalent to solve the following $N$ optimization problems

$$
\begin{aligned}
\max _{\gamma_{k+n}^{i}} & \tilde{\mathbf{H}}_{k+n}^{\prime} \tilde{\mathbf{R}}_{k+n}^{+} \tilde{\mathbf{H}}_{k+n} \quad \text { for } n=1, \ldots, N, \\
\text { subject to } & \sum_{i=1}^{L} \gamma_{k+n}^{i}=m_{k+n}, \\
& \gamma_{k+n}^{i} \in\{0,1\}, i=1,2, \ldots, L .
\end{aligned}
$$

All of $N$ optimization problems have an optimal solution respectively.

Moreover, for the problem (20), we consider minimizing $\mathbf{P}_{k+n \mid k+n}$ and $\mathbf{P}_{k+n+1 \mid k+n+1}$ respectively. Both of them have a recursive decomposition similar to that of minimizing $\mathbf{P}_{k+N \mid k+N}$ for the problem (20). Thus, we have

$$
\begin{aligned}
\min _{\gamma_{k+s}^{i}} & \mathbf{P}_{k+n \mid k+n}\left(\gamma_{k+1}^{1}, \ldots, \gamma_{k+1}^{L}, \ldots, \gamma_{k+n}^{1}, \ldots, \gamma_{k+n}^{L}\right) \\
\text { subject to } & \sum_{i=1}^{L} \gamma_{k+s}^{i}=m_{k+s}, s=1, \ldots, n, \\
& \gamma_{k+s}^{i} \in\{0,1\}, i=1,2, \ldots, L, s=1, \ldots, n,
\end{aligned}
$$


and

$$
\begin{aligned}
\min _{\gamma_{k+s}^{i}} & \mathbf{P}_{k+n+1 \mid k+n+1}\left(\gamma_{k+1}^{1}, \ldots, \gamma_{k+1}^{L}, \ldots, \gamma_{k+n+1}^{1}, \ldots, \gamma_{k+n+1}^{L}\right) \\
\text { subject to } & \sum_{i=1}^{L} \gamma_{k+s}^{i}=m_{k+s}, s=1, \ldots, n+1, \\
& \gamma_{k+s}^{i} \in\{0,1\}, i=1,2, \ldots, L, s=1, \ldots, n+1,
\end{aligned}
$$

have the same optimal solutions $\left(\gamma_{k+s}^{i}\right)^{*}, i=1, \ldots, L, s=1, \ldots, n$ respectively. By Lemma 3.2, the problem (20) is also equivalent to solving the problem (23). Therefore, if the primal sensor selection problem (20) has an optimal solutions, both the problem (20) and the problem (23) can be equivalently transformed to solve the problem (28).

\section{The derivation of the problem (49)}

By $\left(\gamma_{k+n}^{i}\right)^{2}=\gamma_{k+n}^{i}$, the problem (48) is equivalent to

$$
\begin{aligned}
\min _{\gamma_{k+n}^{i}} & \gamma^{\prime} \mathbf{B} \gamma \\
\text { subject to } & \gamma^{\prime} \operatorname{diag}\left(\mathbf{a}_{p}\right) \gamma \unrhd b_{p}, p=1, \ldots, P, \\
& \gamma_{k+n}^{i} \in\{0,1\}, i=1,2, \ldots, L, n=1, \ldots, N,
\end{aligned}
$$

where $\gamma$ is defined in (33); $\operatorname{diag}\left(\mathbf{a}_{p}\right)$ and $\mathbf{B}=\operatorname{diag}\left(\omega_{1} \mathbf{B}_{k+1}, \ldots, \omega_{N} \mathbf{B}_{k+N}\right)$ are diagonal matrix and diagonal block matrix respectively. If we let $\tau_{k+n}^{i}=2 \gamma_{k+n}^{i}-1$ and denote by $\tau_{k+n} \triangleq\left(\tau_{k+n}^{1}, \ldots, \tau_{k+n}^{L}\right)^{\prime}$ and $\tau \triangleq\left(\tau_{k+1}^{\prime}, \ldots, \tau_{k+N}^{\prime}\right)^{\prime}$, then the problem is equivalent to

$$
\min _{\tau_{k+n}^{i}} \frac{1}{4}(\tau+\mathbf{1})^{\prime} \mathbf{B}(\tau+\mathbf{1})
$$

$$
\begin{array}{ll}
\text { subject to } \quad & \frac{1}{4}(\tau+\mathbf{1})^{\prime} \operatorname{diag}\left(\mathbf{a}_{p}\right)(\tau+\mathbf{1}) \unrhd b_{p}, p=1, \ldots, P, \\
& \left(\tau_{k+n}^{i}\right)^{2}=1, i=1,2, \ldots, L, n=1, \ldots, N,
\end{array}
$$

where $\mathbf{1}$ is a 1 vector with compatible dimensions. Moreover, it is equivalent to

$$
\begin{array}{cl}
\min _{\tau_{k+n}^{i}, t} & (\tau+\mathbf{1})^{\prime} \mathbf{B}(\tau+\mathbf{1}) \\
& =\left(\tau_{k+n}^{\prime} t\right)\left(\begin{array}{cc}
\mathbf{B} & \mathbf{B} \mathbf{1} \\
\mathbf{1}^{\prime} \mathbf{B} & 0
\end{array}\right)\left(\begin{array}{c}
\tau_{k+n} \\
t
\end{array}\right) \\
\text { subject to } & \left(\tau^{\prime} t\right)\left(\begin{array}{cc}
\operatorname{diag}\left(\mathbf{a}_{p}\right) & \mathbf{1} \\
\mathbf{1}^{\prime} & 0
\end{array}\right)\left(\begin{array}{c}
\tau \\
t
\end{array}\right) \unrhd 4 b_{p}-\mathbf{1}^{\prime} \operatorname{diag}\left(\mathbf{a}_{p}\right) \mathbf{1}, p=1, \ldots, P, \\
& \left(\tau_{k+n}^{i}\right)^{2}=1, i=1,2, \ldots, L, n=1, \ldots, N, \\
& t^{2}=1 .
\end{array}
$$


Problem (67) is equivalent to (68) in the sense: if $\left(\tau^{*}, t^{*}\right)$ is the optimal solution to (68), then $\tau^{*}$ (respectively $-\tau^{*}$ ) is an optimal solution to (67) when $t^{*}=1$ (respectively $t^{*}=-1$ ). Moreover, the problem is equivalent to

$$
\begin{aligned}
& \min _{\tau, t} \quad\left(\tau^{\prime} t\right) \mathbf{C}\left(\begin{array}{c}
\tau \\
t
\end{array}\right) \\
& \text { subject to } \quad\left(\begin{array}{ll}
\tau^{\prime} & t
\end{array}\right) \mathbf{E}_{0}\left(\begin{array}{c}
\tau \\
t
\end{array}\right) \unrhd\left(4 b_{p}-\mathbf{1}^{\prime} \operatorname{diag}\left(\mathbf{a}_{p}\right) \mathbf{1}\right), \quad p=1, \ldots, P, \\
& \left(\begin{array}{ll}
\tau^{\prime} & t
\end{array}\right) \mathbf{E}_{s}\left(\begin{array}{c}
\tau \\
t
\end{array}\right)=1, s=1, \ldots,(N L+1),
\end{aligned}
$$

where

$$
\begin{gathered}
\mathbf{C}=\left(\begin{array}{cc}
\mathbf{B} & \mathbf{B} \mathbf{1} \\
\mathbf{1}^{\prime} \mathbf{B} & 0
\end{array}\right) \\
\mathbf{E}_{0}=\left(\begin{array}{cc}
\operatorname{diag}\left(\mathbf{a}_{p}\right) & \mathbf{1} \\
\mathbf{1}^{\prime} & 0
\end{array}\right)
\end{gathered}
$$

$\mathbf{E}_{s}$ is a matrix with $s$-row and $s$-column $\mathbf{E}_{s}(s, s)=1$, others equal 0 , for $s=1, \ldots,(N L+1)$. By introducing a new variable $\mathbf{X}=\left(\tau^{\prime} t\right)^{\prime}\left(\tau^{\prime} t\right)$ and removing the constraint $\operatorname{rank}(\mathbf{X})=1$, the problem (69) can be relaxed to the problem (49).

\section{Acknowledgment}

We would like to thank Yunmin Zhu for his helpful suggestions that greatly improved the quality of this paper.

\section{References}

[1] A. O. Hero, D. A. Castañón, D. Cochran, and K. Kastella, Foundations and applications of sensor management. New York: Springer, 2008.

[2] A. O. Hero and D. Cochran, "Sensor management: Past, present, and future," IEEE Sensors Journal, vol. 11, pp. 3064-3075, December 2011.

[3] S. Joshi and S. Boyd, "Sensor selection via convex optimization," IEEE Transactions on Signal Processing, vol. 57, pp. 451-462, February 2009. 
[4] Y. Mo, R. Ambrosino, and B. Sinopoli, "Sensor selection strategies for state estimation in energy constrained wireless sensor networks," Automatica, vol. 47, pp. 1330-1338, 2011.

[5] G. Zhang, S. Ferrari, and M. Qian, "An information roadmap method for robotic sensor path planning," Journal of Intelligent \& Robotic Systems, vol. 56, no. 1, pp. 69-98, 2009.

[6] F. Zhao and L. Guibas, Wireless Sensor Networks: An Information Processing Approach. San Francisco, CA: Morgan Kaufmann, 2004.

[7] V. Gupta, T. H. Chung, B. Hassibi, and R. M. Murray, "On a stochastic sensor selection algorithm with applications in sensor scheduling and sensor coverage," Automatica, vol. 42, no. 2, pp. 251-260, 2006.

[8] S. Jiang, R. Kumar, and H. E. Garcia, "Optimal sensor selection for discrete-event systems with partial observation," IEEE Transactions on Automatic Control, vol. 48, no. 3, pp. 369-381, 2003.

[9] M. L. Hernandez, T. Kirubarajan, and Y. Bar-Shalom, "Multisensor resource deployment using posterior cramer-rao bounds," IEEE Transactions on Aerospace and Electronic Systems, vol. 40, no. 2, pp. 399-416, 2004.

[10] R. Tharmarasa, T. Kirubarajan, M. L. Hernandez, and A. Sinha, "PCRLB-based multisensor array management for multitarget tracking," IEEE Transaction on Aerospace and Electronic Systems, vol. 43, no. 2, pp. 539-555, 2007.

[11] T. Zhao and A. Nehorai, "Information-driven distributed maximum likelihood estimation based on gauss-newton method in wireless sensor networks," IEEE Transactions on Signal Processing, vol. 55, pp. 4669-4682, September 2007.

[12] J. Denzler and C. M. Brown, "Information theoretic sensor data selection for active object recognition and state estimation," IEEE Transactions on Pattern Analysis and Machine Intelligence, vol. 24, no. 2, pp. $145-157,2002$.

[13] I. Mark P. Kolba, Student Member and L. M. Collins, "Information-based sensor management in the presence of uncertainty," IEEE Transactions on Signal Processing, vol. 55, pp. 2731-2735, June 2007.

[14] C. M. Kreucher, A. O. Hero, K. D. Kastella, and M. R. Morelande, "An information-based approach to sensor management in large dynamic networks," Proceedings of the IEEE, vol. 95, pp. 978-999, May 2007.

[15] C. Kreucher, K. Kastella, and A. O.Hero, "Sensor management using an active sensing approach," Signal Processing, vol. 85, no. 3, pp. 607-624, 2005.

[16] E. Masazade, R. Niu, and P. K. Varshney, "Dynamic bit allocation for object tracking in wireless sensor networks," IEEE Transactions on Signal Processing, vol. 60, pp. 5048-5063, October 2012. 
[17] J. L. Williams, J. W. Fisher, and A. S. Willsky, "Approximate dynamic programming for communication-constrained sensor network management," IEEE Transactions on Signal Processing, vol. 55, no. 8, pp. 4300-4311, 2007.

[18] G. M. Hoffmann and C. J. Tomlin, "Mobile sensor network control using mutual information methods and particle filters," IEEE Transactions on Automatic Control, vol. 55, pp. 32-47, January 2010.

[19] N. Xiong and P. Svensson, "Multi-sensor management for information fusion: issues and approaches," Information Fusion, vol. 3, no. 2, pp. 163-186, 2002.

[20] S. Boyd and L. Vandenberghe, Convex Optimization. Cambridge University Press, 2004.

[21] Y. Bar-Shalom, H. Chen, and M. Mallick, "One-step solution for the multistep out-of-sequence measurement problem in tracking," IEEE Transactions on Aerospace and Electronic Systems, vol. 40, pp. 27-37, January 2004.

[22] Y. Zhu, J. Zhou, X. Shen, E. Song, and Y. Luo, Networked Multisensor Decision and Estimation Fusion: Based on Advanced Mathematical Methods. CRC Press, 2012.

[23] A. Ben-Israel and T. N. E. Greville, Generalized inverses: theory and applications. New York: John Wiley, second ed., 2003.

[24] Y. Bar-Shalom and X. Li, Multitarget-Multisensor Tracking: Principles and Techniques. Storrs, CT: YBS Publishing, 1995.

[25] Z.-Q. Luo, W.-K. Ma, A. M.-C. So, Y. Ye, and S. Zhang, "Semidefinite relaxation of quadratic optimization problems," IEEE Signal Processing Magazine, vol. 27, pp. 20-34, May 2010. 


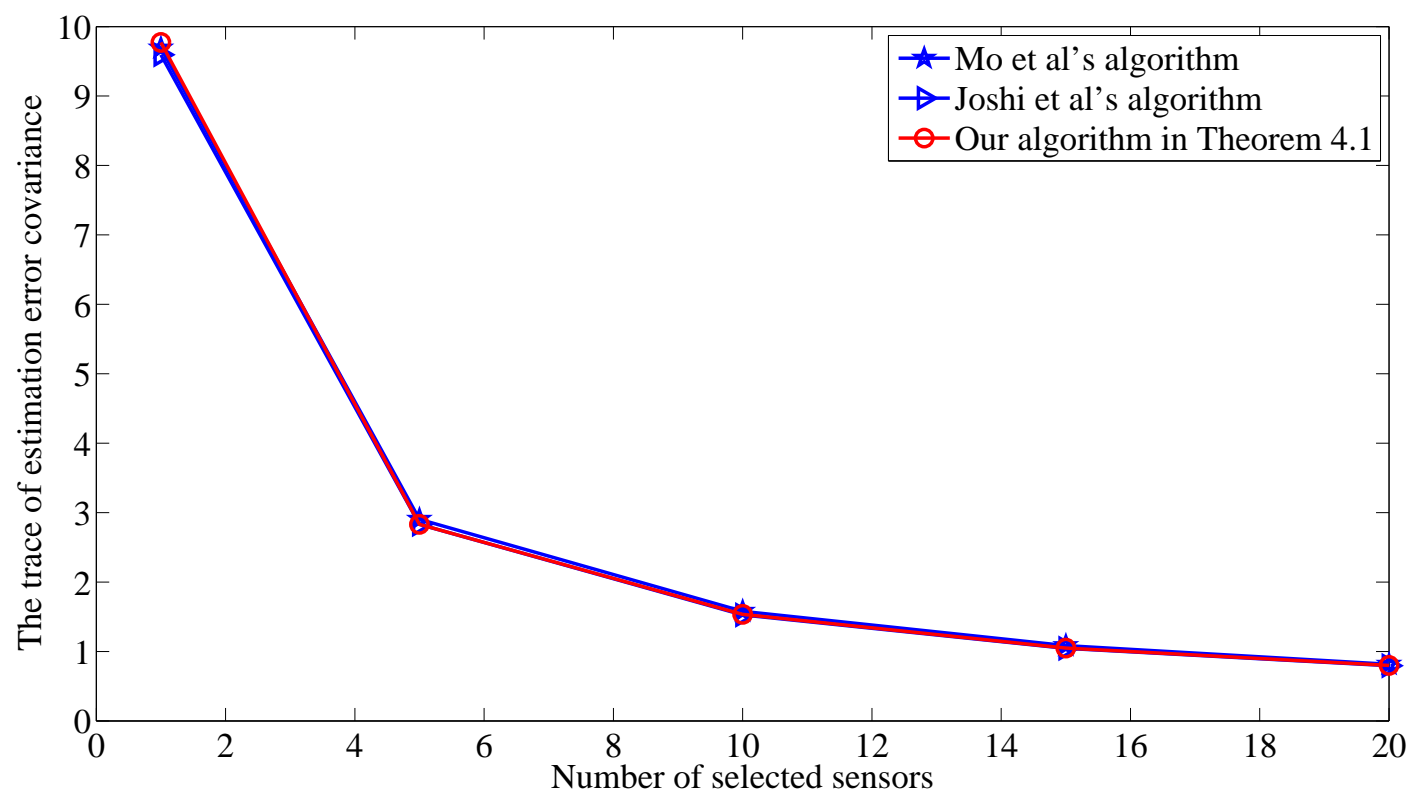

Figure 1: The traces of the estimation error covariance are plotted as a function of number of selected sensors.

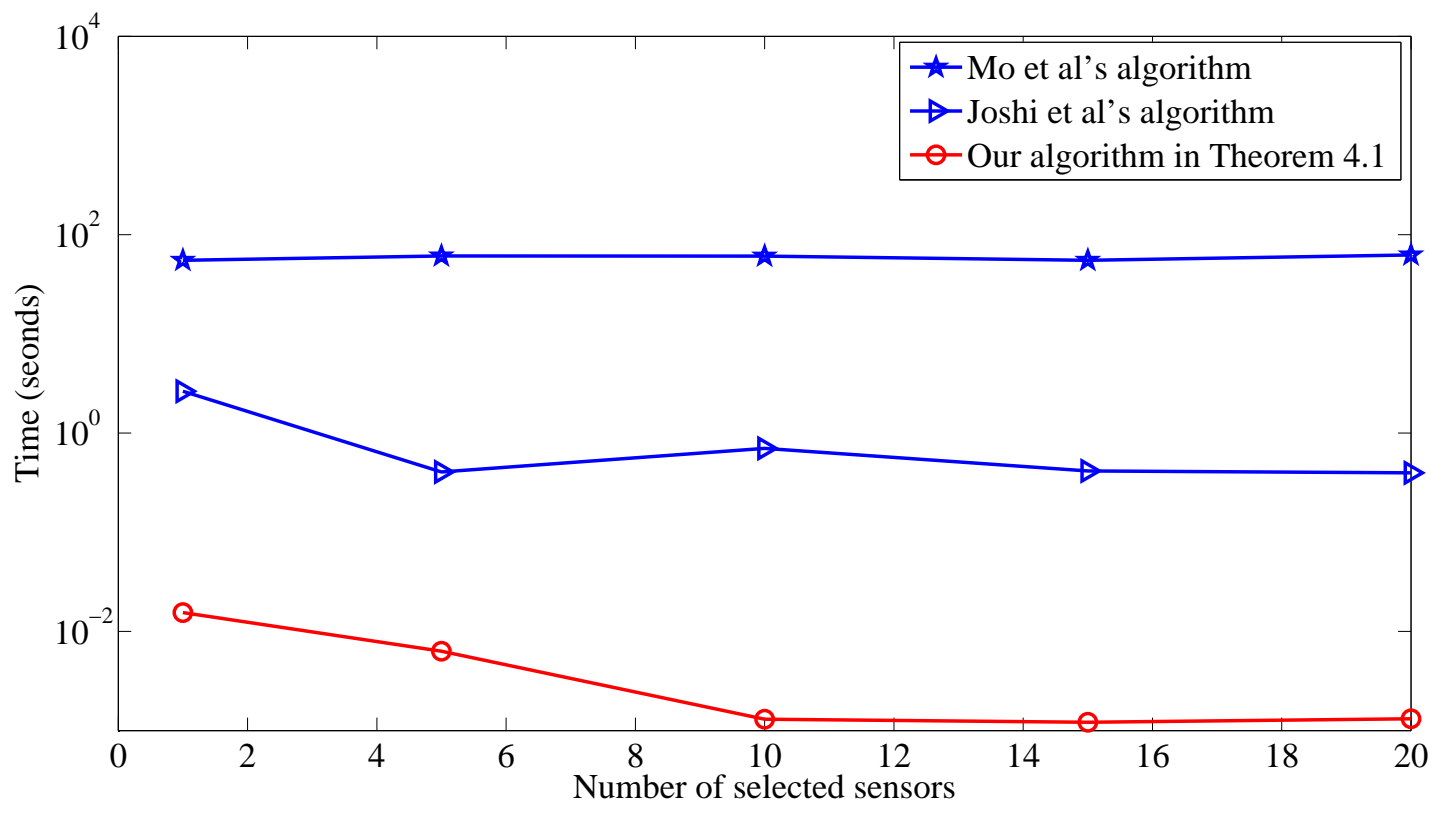

Figure 2: The cpu times are plotted as a function of number of selected sensors. 


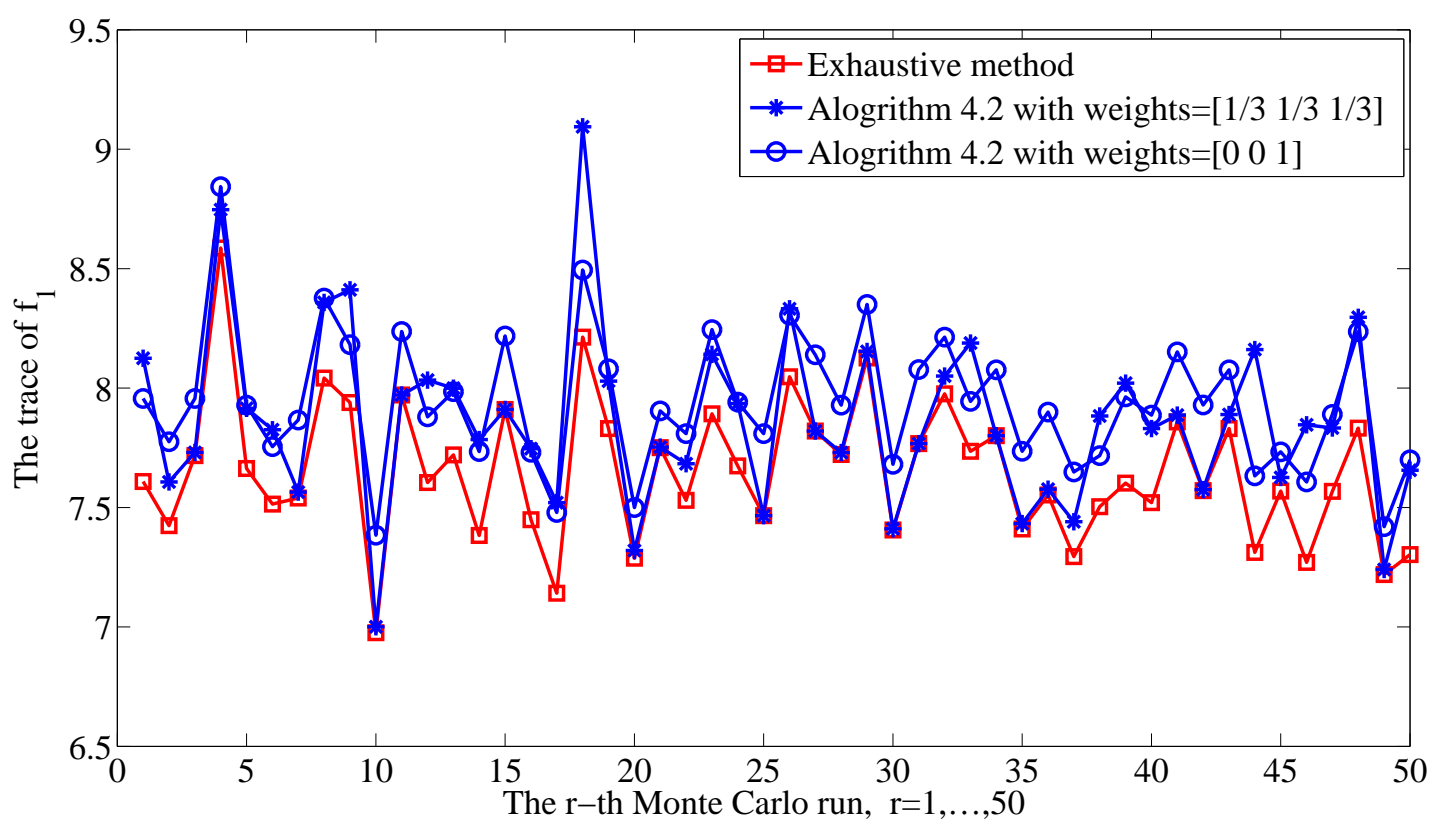

Figure 3: The traces of the final estimation error covariance for 50 Monte Carlo runs.

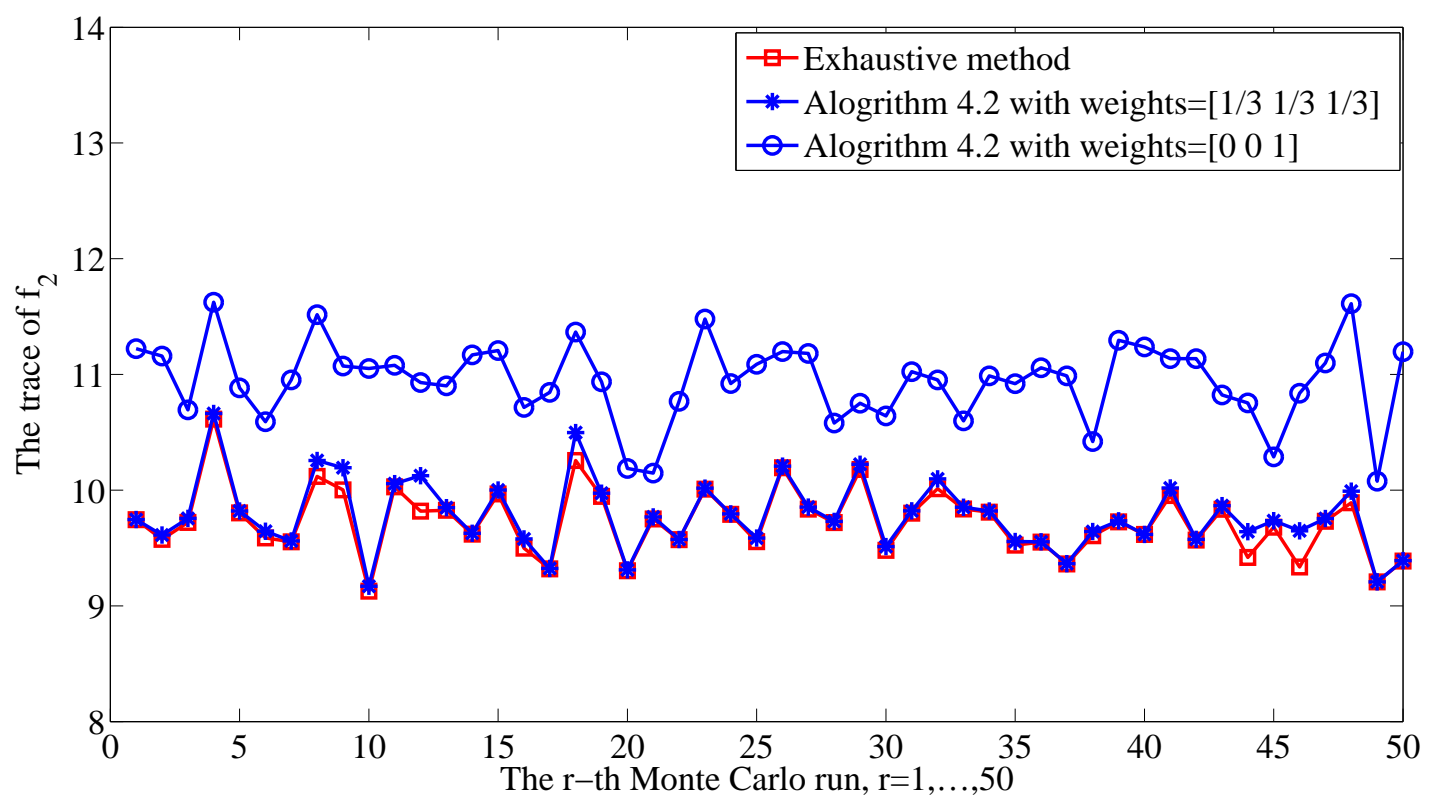

Figure 4: The traces of the average estimation error covariance in 50 Monte Carlo runs. 


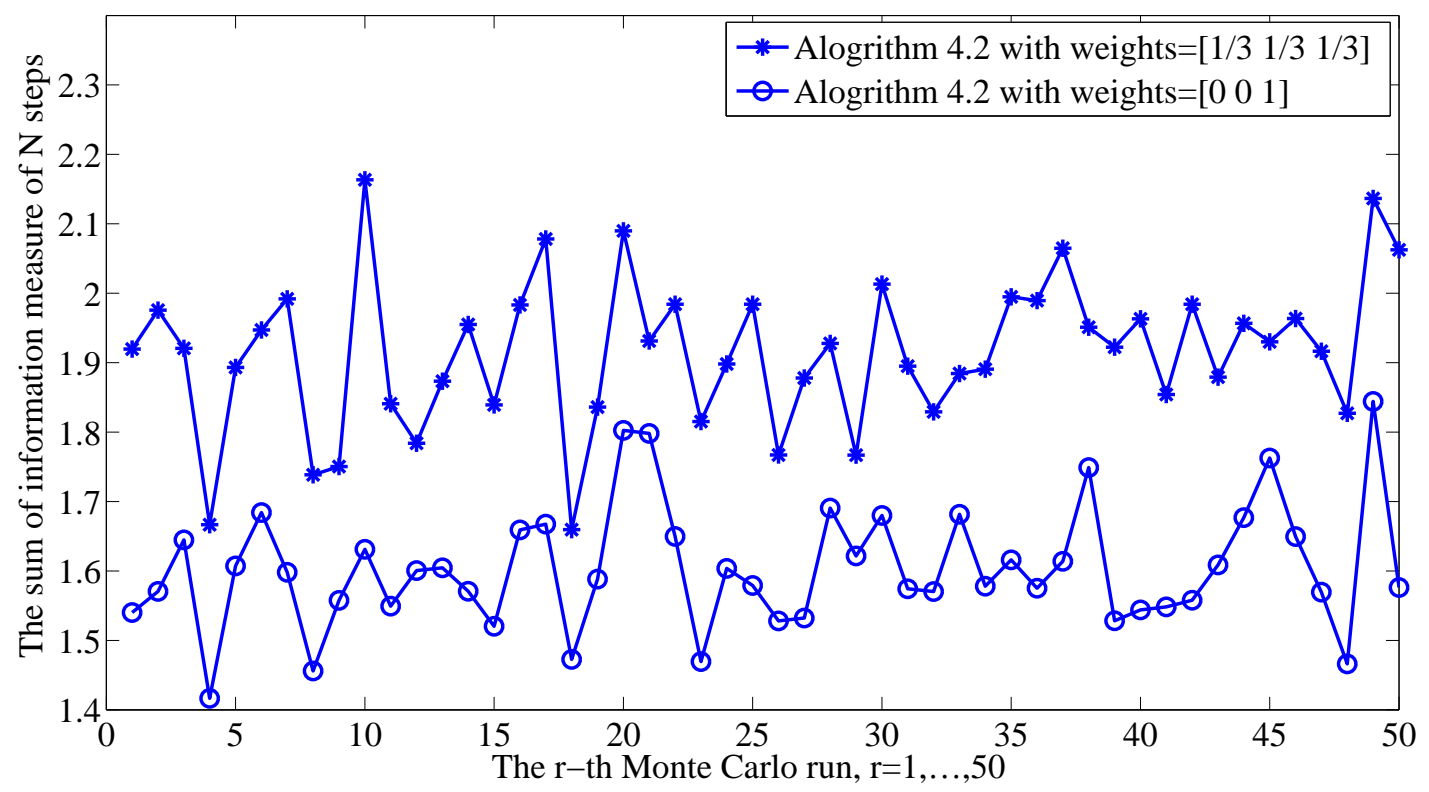

Figure 5: The sum of information measures of $N$ time steps for 50 Monte Carlo runs

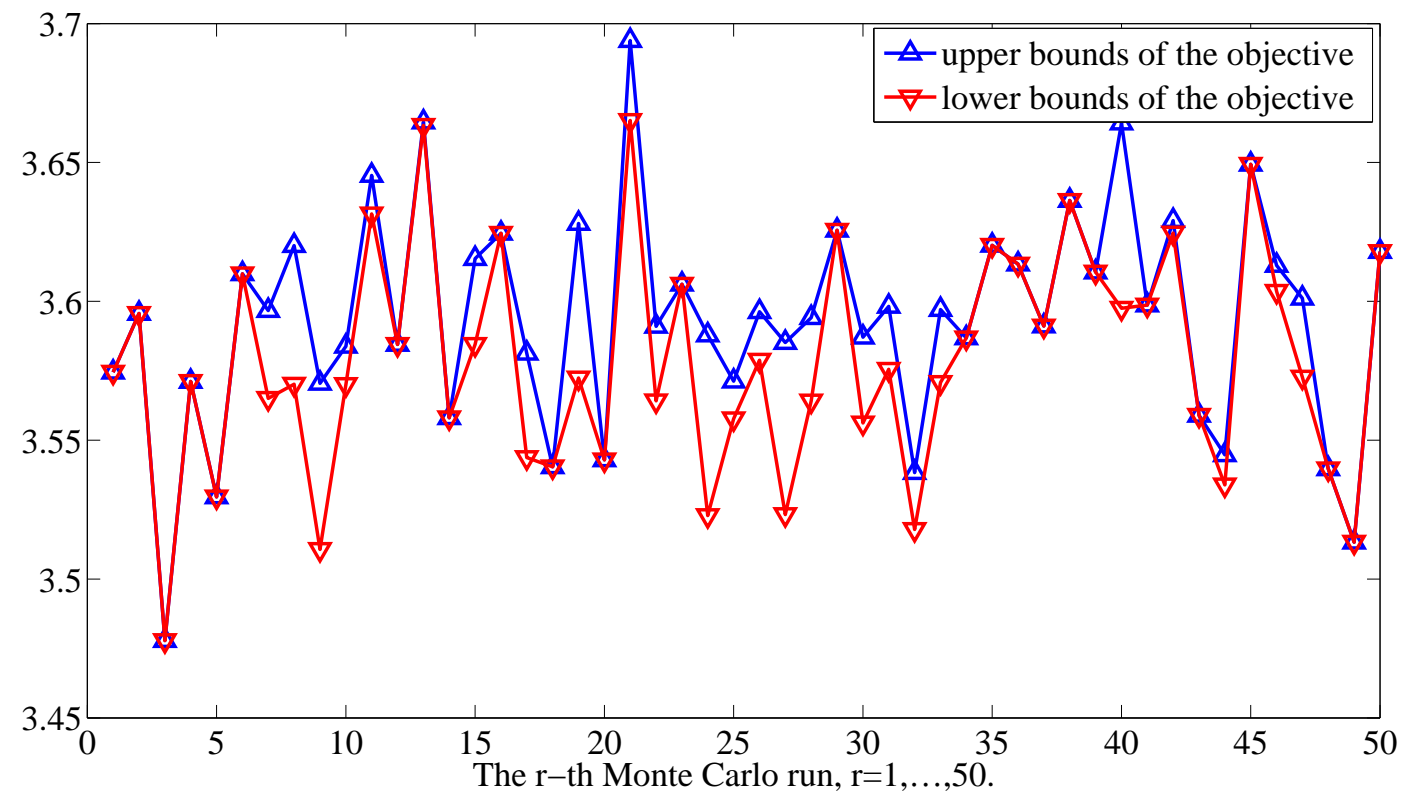

Figure 6: The upper bound and lower bound of the objective function of the optimization problem (37) for 50 Monte Carlo runs. 


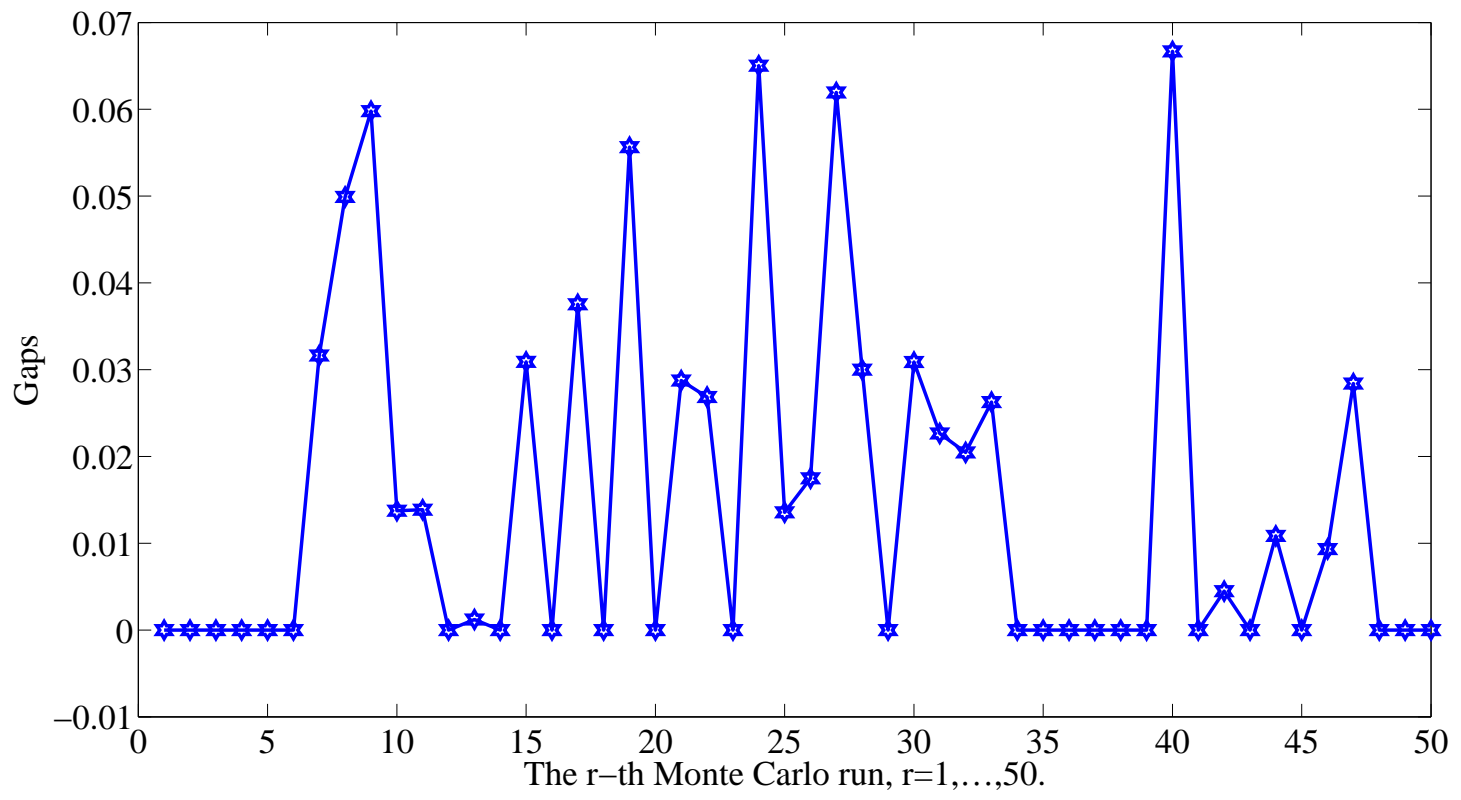

Figure 7: The gaps, i.e. the upper bounds minus the lower bounds shown in Figure6

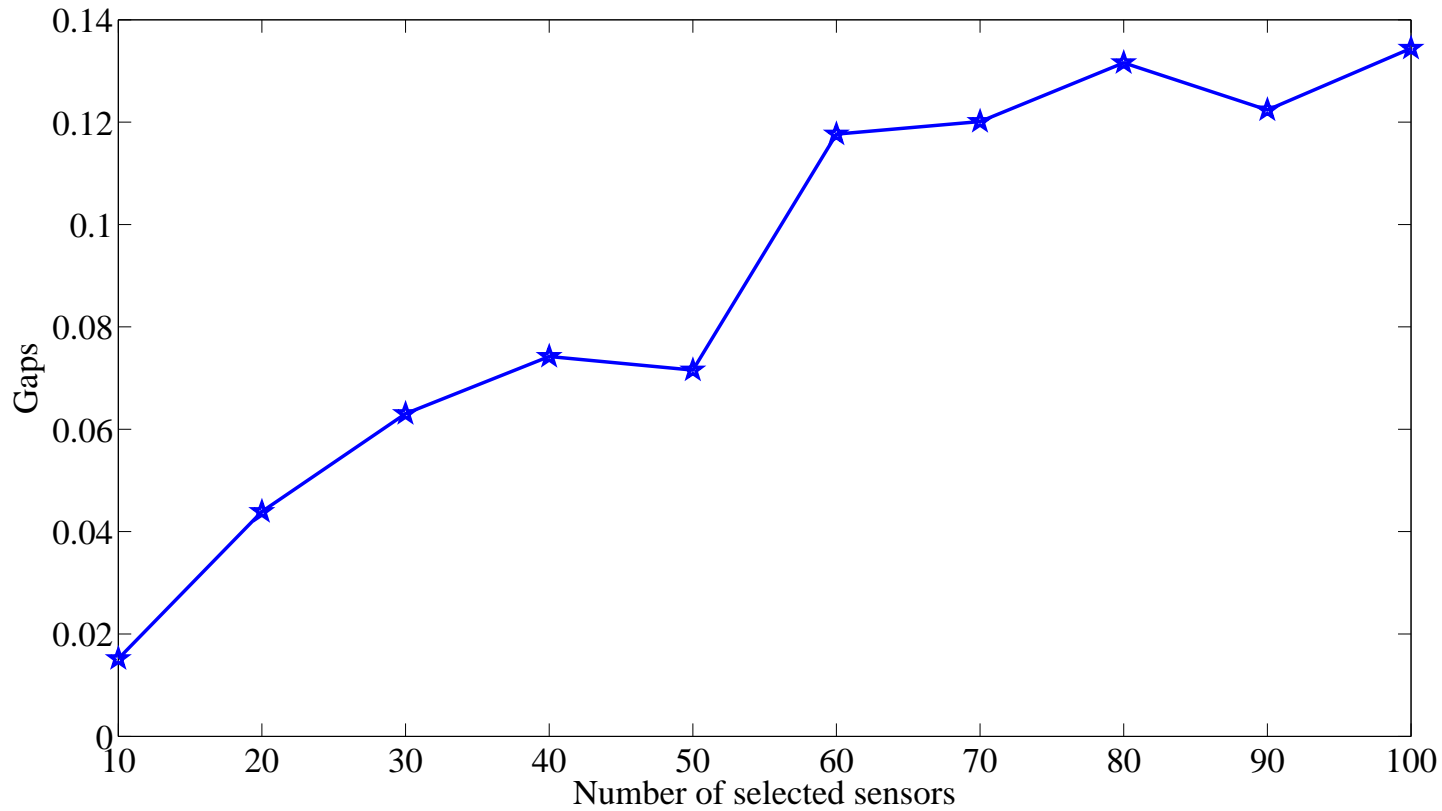

Figure 8: The average gaps based on 50 Monte Carlo runs are plotted as a function of number of selected sensors. 


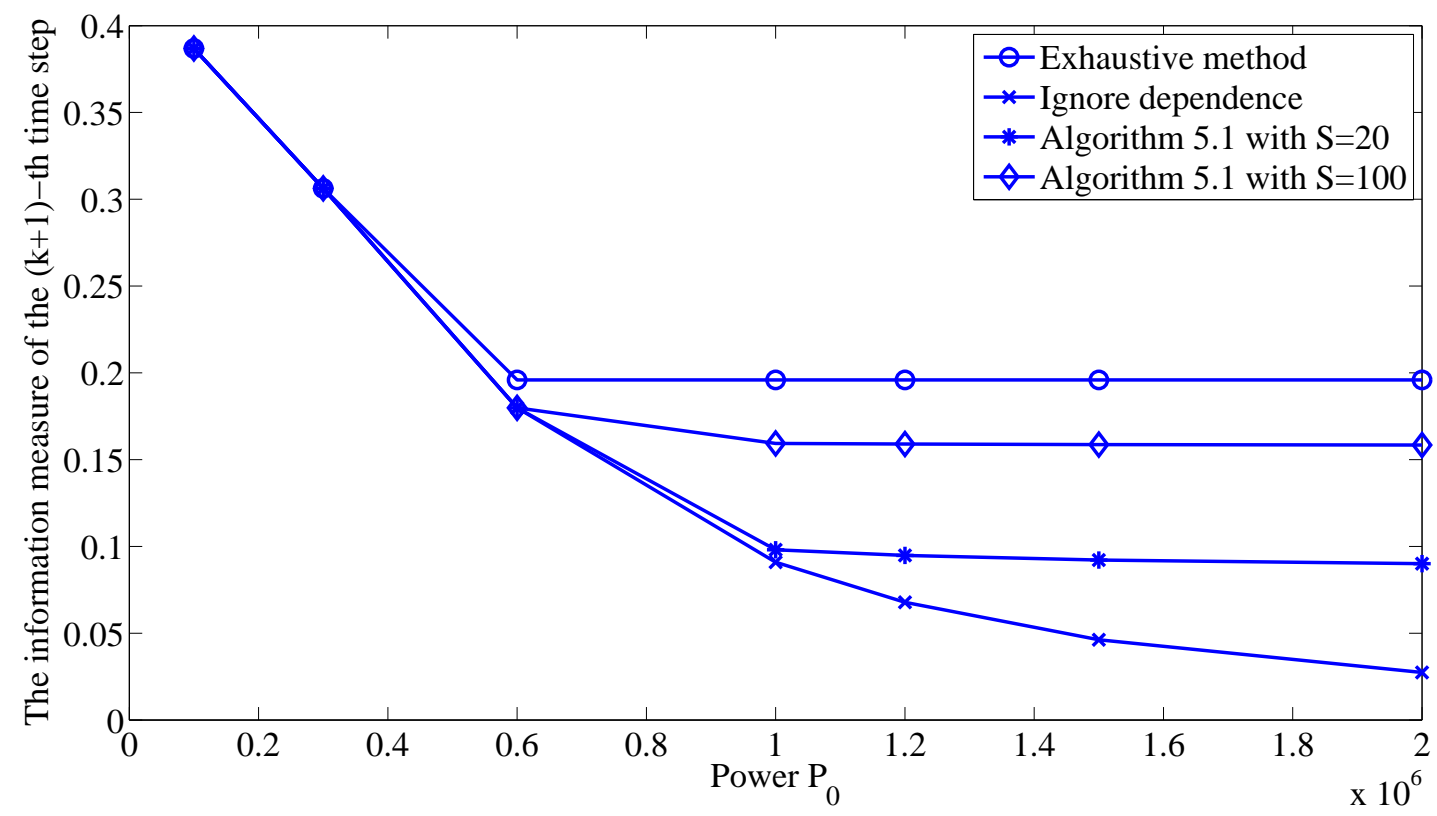

Figure 9: Information measure based on the selected sensors at the $(k+1)$-th time step from weak to strong signal power of the jammer (from weak to strong correlation between sensors).

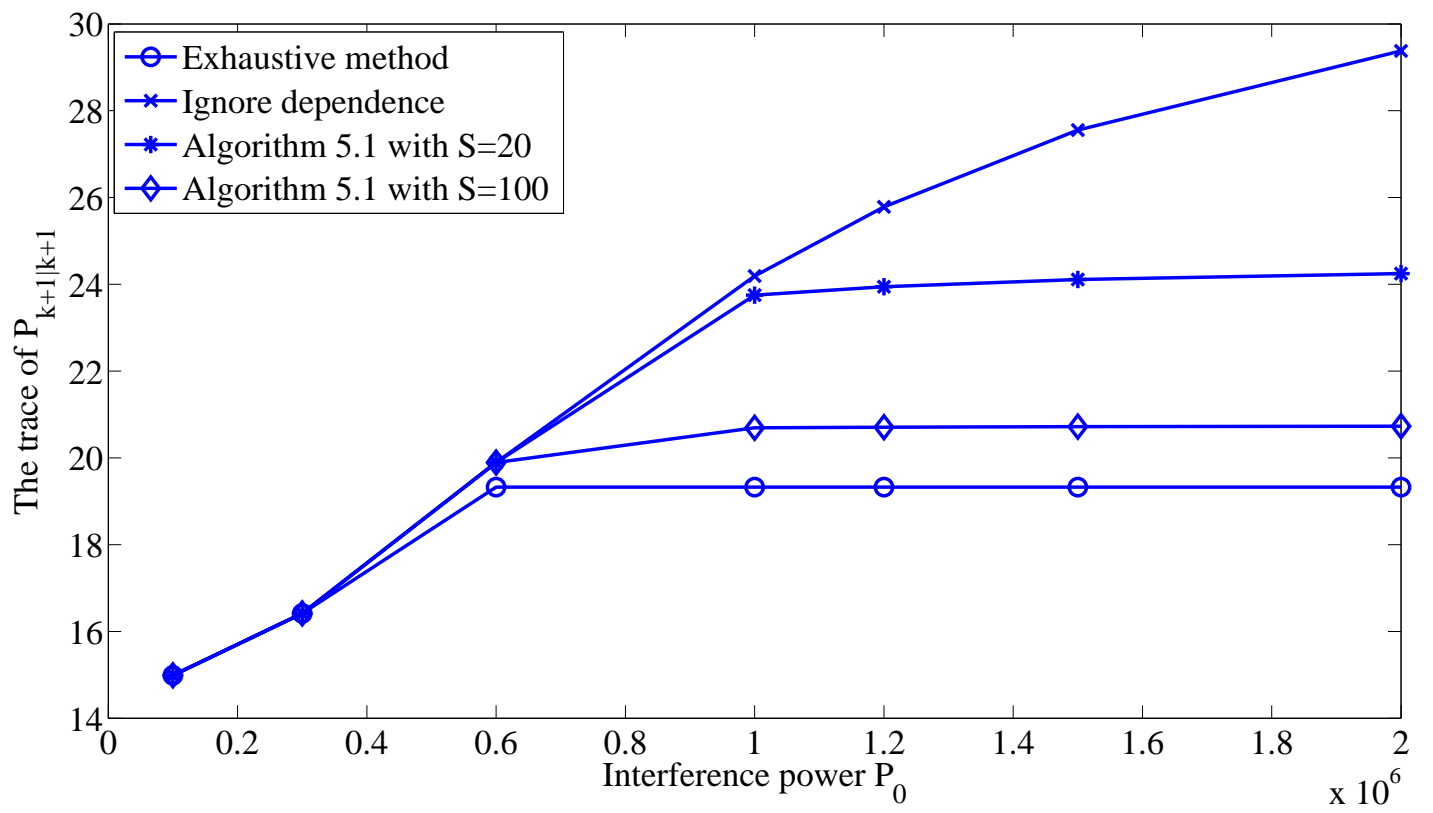

Figure 10: The trace of estimation error covariance at the $(k+1)$-th time step from weak to strong signal power of the jammer (from weak to strong correlation between sensors). 


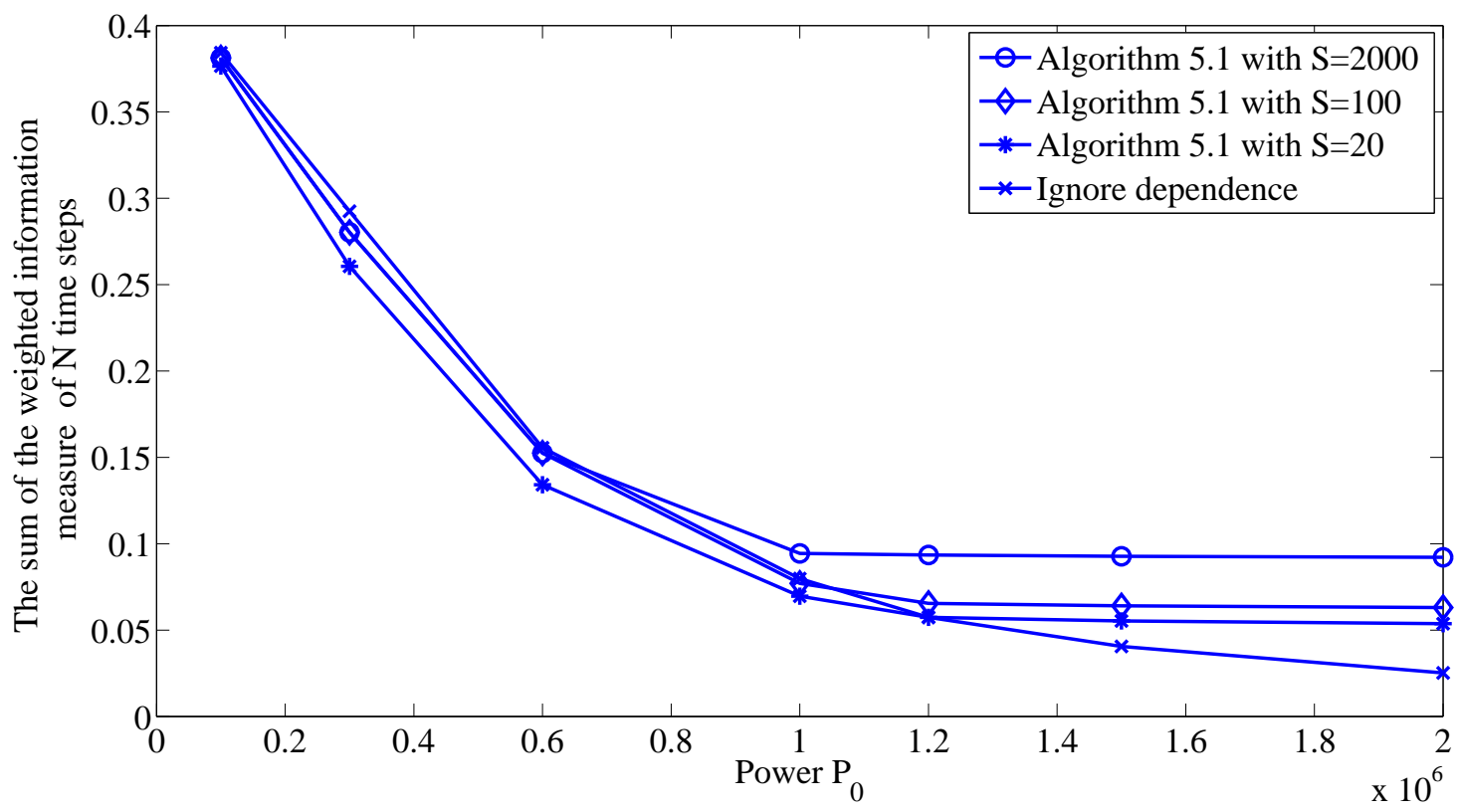

Figure 11: The sum of the weighted information measures of $N$ time steps from weak to strong signal power of the jammer (from weak to strong correlation between sensors).

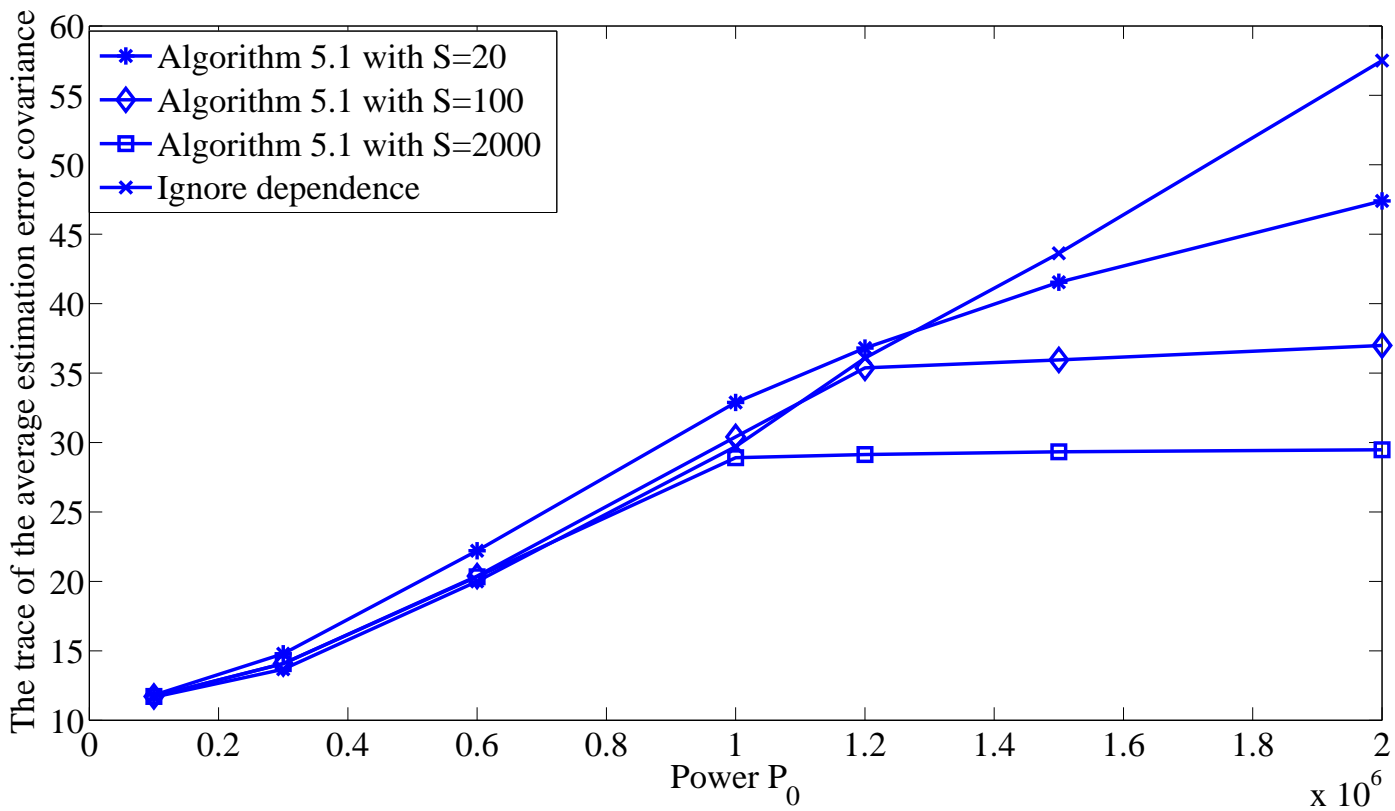

Figure 12: The trace of the average estimation error covariance of $N$ time steps from weak to strong signal power of the jammer (from weak to strong correlation between sensors). 


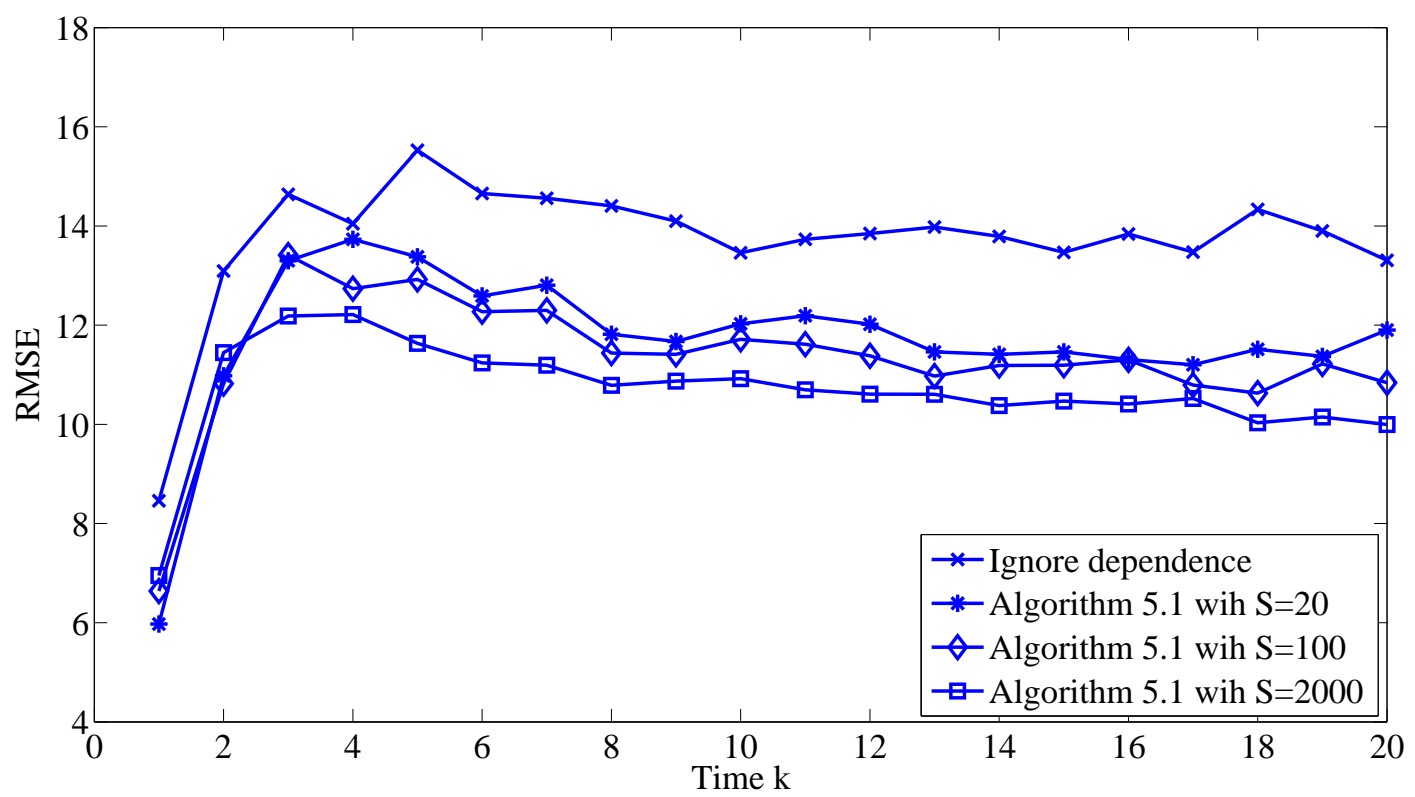

Figure 13: RMSE of the state estimates based on 200 Monte Carlo runs.

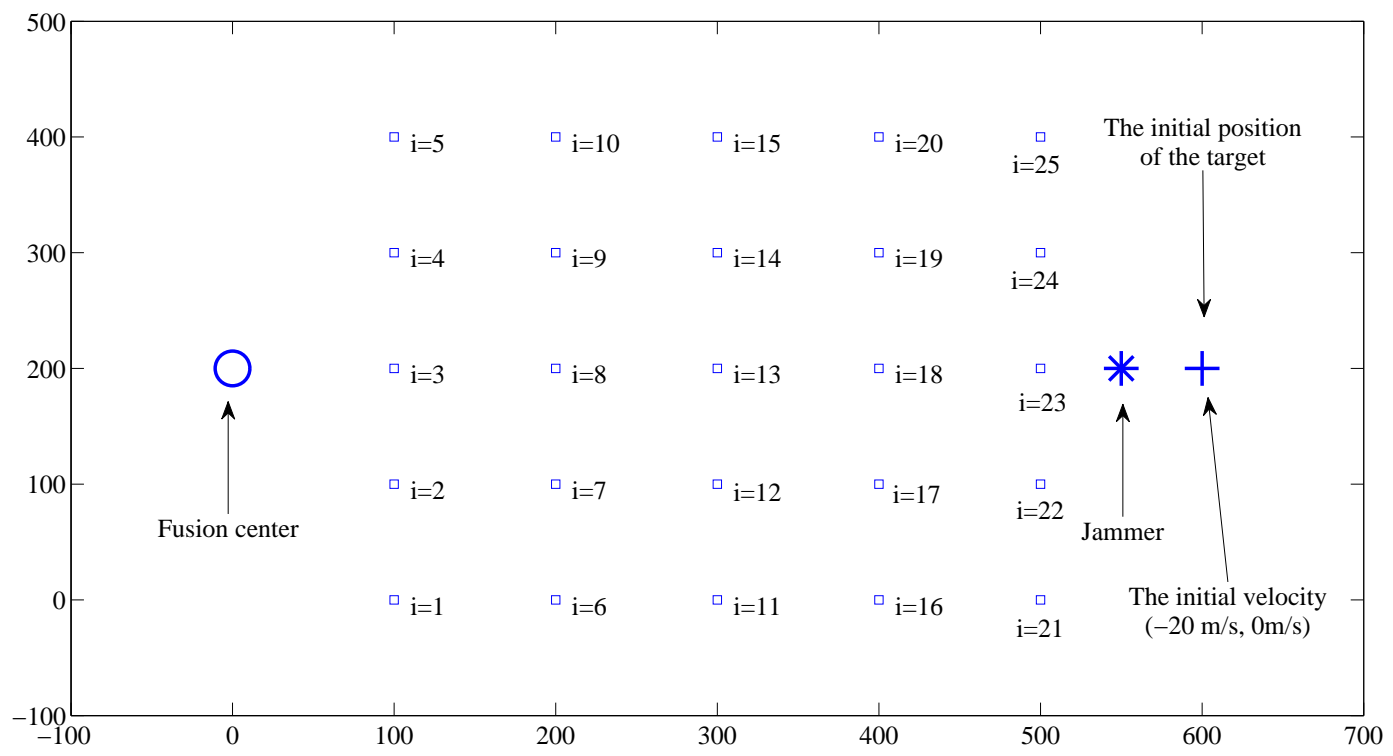

Figure 14: The sensor network with a jammer 